\title{
KAZHDAN-LUSZTIG CELLS AND THE MURPHY BASIS
}

\author{
MEINOLF GECK
}

\begin{abstract}
Let $\mathcal{H}$ be the Iwahori-Hecke algebra associated with $\mathfrak{S}_{n}$, the symmetric group on $n$ symbols. This algebra has two important bases: the Kazhdan-Lusztig basis and the Murphy basis. While the former admits a deep geometric interpretation, the latter leads to a purely combinatorial construction of the representations of $\mathcal{H}$, including the Dipper-James theory of Specht modules. In this paper, we establish a precise connection between the two bases, allowing us to give, for the first time, purely algebraic proofs for a number of fundamental properties of the Kazhdan-Lusztig basis and Lusztig's results on the a-function.
\end{abstract}

\section{INTRODUCTION}

Let $W$ be a finite or affine Weyl group and $\mathcal{H}$ be the associated generic Iwahori-Hecke algebra. By definition, $\mathcal{H}$ is equipped with a standard basis usually denoted by $\left\{T_{w} \mid w \in W\right\}$. In a fundamental paper, Kazhdan and Lusztig 10. constructed a new basis $\left\{C_{w} \mid w \in W\right\}$ and used this, among other applications, to define representations of $\mathcal{H}$ endowed with canonical bases. While that construction is completely elementary, it has deep connections with the geometry of flag varieties and the representation theory of Lie algebras and groups of Lie type. An excellent account of these connections is given in Lusztig's survey [16].

One of the main consequences of the geometric interpretation are certain "positivity properties" for which no elementary proofs have ever been found. In turn, these positivity properties allowed Lusztig to establish a number of fundamental properties of the basis $\left\{C_{w}\right\}$, which are concisely summarized in a list of 15 items $(\mathrm{P} 1)-(\mathrm{P} 15)$ in [17, 14.2]. For example, one application of these properties is the construction of a "canonical" isomorphism from $\mathcal{H}$ onto the group algebra of $W$ (when $W$ is finite); see Lusztig [12].

This paper arose from the desire to give elementary, purely algebraic proofs for $(\mathrm{P} 1)-(\mathrm{P} 15)$ in the case where $W$ is the symmetric group $\mathfrak{S}_{n}$. This goal will be achieved in Secetion 5; we will also prove a tiny piece of "positivity" in this case, namely, the fact that the structure constants of Lusztig's ring $J$ are 0 or 1 . The key idea of our approach is to relate the basis $\left\{C_{w}\right\}$ to the basis constructed by Murphy 20, 21. The main problem in establishing that relation is that the Murphy basis elements are not directly indexed by the elements of $W=\mathfrak{S}_{n}$. Indeed, those elements are written as $x_{\mathfrak{s t}}$ where

Date: April 6, 2005.

2000 Mathematics Subject Classification. Primary $20 \mathrm{C} 08$. 
$(\mathfrak{s}, \mathfrak{t})$ runs over all pairs of standard $\lambda$-tableaux, for various partitions $\lambda$ of $n$. Now, the Robinson-Schensted correspondence does associate to each element of $\mathfrak{S}_{n}$ a pair of standard tableaux of the same shape, but this works on a purely combinatorial level; we need to relate this to basis elements of $\mathcal{H}$. Eventually, we shall see that the "leading matrix coefficients" introduced by the author [5] provide a bridge to pass from the Kazhdan-Lusztig basis to the Murphy basis of $\mathcal{H}$; see Theorem 4.10] and Corollary 4.11. While the explicit form of the base change seems to be rather complicated, our results are sufficiently fine to enable us to translate combinatorial properties of the Murphy basis into properties of the Kazhdan-Lusztig basis.

This paper is organized as follows. In Section 2, we recall the basic definitions concerning the Kazhdan-Lusztig basis of $\mathcal{H}$, the left cells and the corresponding representations. In Section 3, we consider the orthogonal representations and leading matrix coefficients introduced by the author [5]. These provide an efficient tool for identifying Kazhdan-Lusztig basis elements and irreducible representations occuring as constituents in left cell representations.

In the remaining parts of this paper, we exclusively consider the case where $W=\mathfrak{S}_{n}$ is the symmetric group. In Section 4, we recall the construction of the Murphy basis $\left\{x_{\mathfrak{s t}}\right\}$ of $\mathcal{H}$. One of Murphy's key results is that, for a fixed partition $\lambda$, the submodule $N^{\lambda}$ spanned by all basis elements $x_{\mathfrak{s t}}$, where $(\mathfrak{s}, \mathfrak{t})$ are standard $\mu$-tableaux such that $\lambda \unlhd \mu$, is a two-sided ideal in $\mathcal{H}$. In Theorem 4.10, we show that the image of $N^{\lambda}$ under a certain algebra automorphism is spanned by the Kazhdan-Lusztig basis elements $C_{w}$, where $w$ has a non-zero leading matrix coefficient in a representation labelled by a partition $\mu$ such that $\lambda \unlhd \mu$.

Finally, in Section 5, we discuss the applications to the left cells in $\mathfrak{S}_{n}$. In Theorem 5.1] we characterize the Kazhdan-Lusztig pre-order relation $\leqslant_{\mathcal{L R}}$ in terms of the dominance order on partitions. In Theorem 5.3 we establish Lusztig's "Property (A)" which played a decisive role in [12. In Theorem [5.6. we interprete our results in terms of the Robinson-Schensted correspondence; our approach even yields a new proof for a key step in establishing the fact that the left cells of $\mathfrak{S}_{n}$ are given by the RobinsonSchensted correspondence. To deal with Lusztig's a-function, we rely on the methods developped by Iancu and the author in [7, §4]. These methods show that most of the properties (P1)-(P15) follow from a relatively small set of hypotheses which are easily seen to be satisfied thanks to the link between the Kazhdan-Lusztig basis and the Murphy basis; see Theorem 5.10.

\section{KAZHDAN-LUSZTig CELLS}

Let $W$ be a Coxeter group with (finite) generating set $S$. Until the end of this section, we do not need to assume that $W$ is finite. Let $l: W \rightarrow \mathbb{N}$ be the usual length function with respect to $S$ (where $\mathbb{N}=\{0,1,2, \ldots\}$ ). Let $A=\mathbb{Z}\left[v, v^{-1}\right]$ be the ring of Laurent polynomials in an indeterminate $v$. Let 
$\mathcal{H}=\mathcal{H}_{A}(W, S)$ be the corresponding Iwahori-Hecke algebra. Then $\mathcal{H}$ is free over $A$ with basis $\left\{T_{w} \mid w \in W\right\}$; the multiplication is given by the rule

$$
T_{s} T_{w}=\left\{\begin{array}{cl}
T_{s w} & \text { if } l(s w)=l(w)+1 \\
T_{s w}+\left(v-v^{-1}\right) T_{w} & \text { if } l(s w)=l(w)-1
\end{array}\right.
$$

where $w \in W$ and $s \in S$. For basic properties of $W$ and $\mathcal{H}$, we refer to [8].

2.1. Kazhdan-Lusztig bases. There are two types of Kazhdan-Lusztig bases, denoted by $\left\{C_{w} \mid w \in W\right\}$ and $\left\{C_{w}^{\prime} \mid w \in W\right\}$ in the original article by Kazhdan and Lusztig [10]. In [17, Lusztig writes $c_{w}$ instead of $C_{w}^{\prime}$, but we shall adhere to the older notation here. The precise definitions are as follows.

There is a unique ring involution $A \rightarrow A, a \mapsto \bar{a}$, such that $\bar{v}=v^{-1}$. We can extend this map to a ring involution $j: \mathcal{H} \rightarrow \mathcal{H}$ such that

$$
j\left(\sum_{w \in W} a_{w} T_{w}\right)=\sum_{w \in W} \varepsilon_{w} \bar{a}_{w} T_{w} \quad\left(a_{w} \in A\right),
$$

where we write $\varepsilon_{w}=(-1)^{l(w)}$ for any $w \in W$. Furthermore, there is an $A$-algebra automorphism

$$
\dagger: \mathcal{H} \rightarrow \mathcal{H}, \quad T_{s} \mapsto T_{s}^{\dagger}=-T_{s}^{-1} \quad(s \in S) .
$$

It is easily checked that $j$ and $\dagger$ commute with each other. For any $h \in \mathcal{H}$, we set $\bar{h}:=j(h)^{\dagger}=j\left(h^{\dagger}\right)$. Thus, the map $\mathcal{H} \rightarrow \mathcal{H}, h \mapsto \bar{h}$, is a ring involution such that

$$
\overline{\sum_{w \in W} a_{w} T_{w}}=\sum_{w \in W} \bar{a}_{w} T_{w^{-1}}^{-1} \quad\left(a_{w} \in A\right) .
$$

Now, for each $w \in W$, there exists a unique element $C_{w}^{\prime} \in \mathcal{H}$ such that

$$
\bar{C}_{w}^{\prime}=C_{w}^{\prime} \quad \text { and } \quad C_{w}^{\prime} \equiv T_{w} \quad \bmod \mathcal{H}_{<0},
$$

where $\mathcal{H}_{<0}:=\sum_{w \in W} A_{<0} T_{w}$ and $A_{<0}:=v^{-1} \mathbb{Z}\left[v^{-1}\right]$; see [17, Theorem 5.2]. The elements $\left\{C_{w}^{\prime} \mid w \in W\right\}$ form an $A$-basis of $\mathcal{H}$, and we have

$$
C_{w}^{\prime}=T_{w}+\sum_{\substack{y \in W \\ y<w}} p_{y, w} T_{y}
$$

where $<$ denotes the Bruhat-Chevalley order on $W$ and $p_{y, w} \in A_{<0}$ for all $y<w$ in $W$. It will be technically more convenient to work with the $C$-basis of $\mathcal{H}$. (The reasons can be seen, for example, in [17, Chap. 18].) We set $C_{w}:=\varepsilon_{w} j\left(C_{w}^{\prime}\right)$. Then we have

$$
C_{w}=T_{w}+\sum_{\substack{y \in W \\ y<w}} \varepsilon_{y} \varepsilon_{w} \bar{p}_{y, w} T_{y} \quad \text { for all } w \in W .
$$

As before, one shows that the basis element $C_{w}$ is uniquely determined by the conditions that

$$
\bar{C}_{w}=C_{w} \quad \text { and } \quad C_{w} \equiv T_{w} \quad \bmod \mathcal{H}_{>0},
$$

where $\mathcal{H}_{>0}:=\sum_{w \in W} A_{>0} T_{w}$ and $A_{>0}:=v \mathbb{Z}[v]$. 
2.2. Multiplication rules. For any $x, y \in W$, we write

$$
C_{x}^{\prime} C_{y}^{\prime}=\sum_{z \in W} h_{x, y, z} C_{z}^{\prime} \quad \text { where } h_{x, y, z} \in A
$$

We have the following more explicit formula for $s \in S, y \in W$ (see [17, §6]):

$$
C_{s}^{\prime} C_{y}^{\prime}= \begin{cases}C_{s y}^{\prime}+\sum_{\substack{z \in W \\ s z<z<y}} \mu_{z, y} C_{z}^{\prime} & \text { if } s y>y, \\ \left(v+v^{-1}\right) C_{y}^{\prime} & \text { if } s y<y,\end{cases}
$$

where $\mu_{z, y} \in \mathbb{Z}$ is the coefficient of $v^{-1}$ in $p_{z, y}$; see [17, Cor. 6.5]. Using the relation $C_{w}=\varepsilon_{w} j\left(C_{w}^{\prime}\right)$, we obtain the formula

$$
C_{x} C_{y}=\sum_{z \in W} \varepsilon_{x} \varepsilon_{y} \varepsilon_{z} h_{x, y, z} C_{z} .
$$

Note also that, for $s \in S$, we have $C_{s}^{\prime}=T_{s}+v^{-1} T_{1}$ and $C_{s}=T_{s}-v T_{1}$.

2.3. The Kazhdan-Lusztig pre-orders. As in [17, §8], we write $x \leftarrow \mathcal{L} y$ if there exists some $s \in S$ such that $h_{s, y, x} \neq 0$, that is, $C_{x}^{\prime}$ occurs in $C_{s}^{\prime} C_{y}^{\prime}$ (when expressed in the $C^{\prime}$-basis) or, equivalently, $C_{x}$ occurs in $C_{s} C_{y}$ (when expressed in the $C$-basis). The Kazhdan-Lusztig left pre-order $\leqslant_{\mathcal{L}}$ is the relation on $W$ generated by $\leftarrow \mathcal{L}$, that is, we have $x \leqslant \mathcal{L} y$ if there exists a sequence $x=x_{0}, x_{1}, \ldots, x_{k}=y$ of elements in $W$ such that $x_{i-1} \leftarrow \mathcal{L} x_{i}$ for all $i$. The equivalence relation associated with $\leqslant_{\mathcal{L}}$ will be denoted by $\sim_{\mathcal{L}}$ and the corresponding equivalence classes are called the left cells of $W$.

Similarly, we can define a pre-order $\leqslant \mathcal{R}$ by considering multiplication by $C_{s}^{\prime}$ on the right in the defining relation. The equivalence relation associated with $\leqslant_{\mathcal{R}}$ will be denoted by $\sim_{\mathcal{R}}$ and the corresponding equivalence classes are called the right cells of $W$. We have

$$
x \leqslant_{\mathcal{R}} y \quad \Leftrightarrow \quad x^{-1} \leqslant_{\mathcal{L}} y^{-1} .
$$

This follows by using the antiautomorphism $b: \mathcal{H} \rightarrow \mathcal{H}$ given by $T_{w}^{b}=T_{w^{-1}}$; we have $C_{w}^{b}=C_{w^{-1}}^{\prime}$ and $C_{w}^{b}=C_{w^{-1}}$ for all $w \in W$; see [17, 5.6]. Thus, any statement concerning the left pre-order relation $\leqslant_{\mathcal{L}}$ has an equivalent version for the right pre-order relation $\leqslant_{\mathcal{R}}$, via $b$.

Finally, we define a pre-order $\leqslant \mathcal{L R}_{\mathcal{R}}$ by the condition that $x \leqslant \mathcal{L R}$ y if there exists a sequence $x=x_{0}, x_{1}, \ldots, x_{k}=y$ such that, for each $i \in\{1, \ldots, k\}$, we have $x_{i-1} \leqslant_{\mathcal{L}} x_{i}$ or $x_{i-1} \leqslant_{\mathcal{R}} x_{i}$. The equivalence relation associated with $\leqslant_{\mathcal{L R}}$ will be denoted by $\sim_{\mathcal{L R}}$ and the corresponding equivalence classes are called the two-sided cells of $W$.

2.4. Lusztig's conjectures. For the convenience of the reader, we recall here Lusztig's conjectures (P1)-(P15) from [17, Chap. 14]. (In fact, Lusztig formulates these conjectures in a slightly more general setting, where $\mathcal{H}$ is defined with respect to a weight function $L$ on $W$; but we will not need to discuss that more general situation here.) For a fixed $z \in W$, we set

$$
\mathbf{a}(z):=\min \left\{i \geqslant 0 \mid v^{i} h_{x, y, z} \in \mathbb{Z}[v] \text { for all } x, y \in W\right\} ;
$$


this is Lusztig's function a: $W \rightarrow \mathbb{N}$, introduced in 14. Note that, for infinite $W$, it is not at all clear if $\mathbf{a}(z)<\infty$ for all $z \in W$. However, Lusztig [17. 13.4] conjectures that this is always the case.

Furthermore, we define $\Delta(z) \in \mathbb{Z}$ and $0 \neq n_{z} \in \mathbb{Z}$ by the condition

$$
p_{1, z}=n_{z} v^{-\Delta(z)}+\text { combination of smaller powers of } v \text {; }
$$

note that $\Delta(z) \geqslant 0$. Given $x, y, z \in W$, we define $\gamma_{x, y, z^{-1}} \in \mathbb{Z}$ by

$$
\gamma_{x, y, z^{-1}}=\text { constant term of } v^{\mathbf{a}(z)} h_{x, y, z} \in \mathbb{Z}[v] .
$$

Let

$$
\mathcal{D}:=\{z \in W \mid \mathbf{a}(z)=\Delta(z)\} .
$$

Then Lusztig [17, 14.2] conjectures that the following properties hold.

P1. For any $z \in W$ we have $\mathbf{a}(z) \leqslant \Delta(z)$.

P2. If $d \in \mathcal{D}$ and $x, y \in W$ satisfy $\gamma_{x, y, d} \neq 0$, then $x=y^{-1}$.

P3. If $y \in W$, there exists a unique $d \in \mathcal{D}$ such that $\gamma_{y^{-1}, y, d} \neq 0$.

P4. If $z^{\prime} \leqslant_{\mathcal{L R}} z$ then $\mathbf{a}\left(z^{\prime}\right) \geqslant \mathbf{a}(z)$. Hence, if $z^{\prime} \sim_{\mathcal{L} \mathcal{R}} z$, then $\mathbf{a}(z)=\mathbf{a}\left(z^{\prime}\right)$.

P5. If $d \in \mathcal{D}, y \in W, \gamma_{y^{-1}, y, d} \neq 0$, then $\gamma_{y^{-1}, y, d}=n_{d}= \pm 1$.

P6. If $d \in \mathcal{D}$, then $d^{2}=1$.

P7. For any $x, y, z \in W$, we have $\gamma_{x, y, z}=\gamma_{y, z, x}$.

P8. Let $x, y, z \in W$ be such that $\gamma_{x, y, z} \neq 0$. Then $x \sim_{\mathcal{L}} y^{-1}, y \sim_{\mathcal{L}} z^{-1}$, $z \sim_{\mathcal{L}} x^{-1}$.

P9. If $z^{\prime} \leqslant \mathcal{L} z$ and $\mathbf{a}\left(z^{\prime}\right)=\mathbf{a}(z)$, then $z^{\prime} \sim_{\mathcal{L}} z$.

P10. If $z^{\prime} \leqslant_{\mathcal{R}} z$ and $\mathbf{a}\left(z^{\prime}\right)=\mathbf{a}(z)$, then $z^{\prime} \sim_{\mathcal{R}} z$.

P11. If $z^{\prime} \leqslant \mathcal{L R} z$ and $\mathbf{a}\left(z^{\prime}\right)=\mathbf{a}(z)$, then $z^{\prime} \sim_{\mathcal{L R}} z$.

P12. Let $I \subset S$ and $W_{I}$ be the parabolic subgroup generated by $I$. If $y \in$ $W_{I}$, then $\mathbf{a}(y)$ computed in terms of $W_{I}$ is equal to $\mathbf{a}(y)$ computed in terms of $W$.

P13. Any left cell $\mathfrak{C}$ of $W$ contains a unique element $d \in \mathcal{D}$. We have $\gamma_{x^{-1}, x, d} \neq 0$ for all $x \in \mathfrak{C}$.

P14. For any $z \in W$, we have $z \sim \mathcal{L R} z^{-1}$.

P15. Let $\breve{v}$ be a second indeterminate and let $\breve{h}_{x, y, z} \in \mathbb{Z}\left[\breve{v}, \breve{v}^{-1}\right]$ be obtained from $h_{x, y, z}$ by the substitution $v \mapsto \breve{v}$. If $x, x^{\prime}, y, w \in W$ satisfy $\mathbf{a}(w)=\mathbf{a}(y)$, then

$$
\sum_{y^{\prime} \in W} \breve{h}_{w, x^{\prime}, y^{\prime}} h_{x, y^{\prime}, y}=\sum_{y^{\prime} \in W} h_{x, w, y^{\prime}} \breve{h}_{y^{\prime}, x^{\prime}, y} .
$$

If $W$ is a finite or affine Weyl group then all these properties hold thanks to a geometric interpretation which yields the following "positivity property":

$$
p_{x, y} \in v^{-1} \mathbb{N}\left[v^{-1}\right] \quad \text { and } \quad h_{x, y, z} \in \mathbb{N}\left[v, v^{-1}\right] \quad(x, y, z \in W) ;
$$

see the discussion of the "split case" by Lusztig [17, Chap. 15] and the references there; see also Springer [22].

Once (P1)-(P15) are known to hold, one can construct the ring $J$ as explained in [17, Chap. 18]. As an abelian group, $J$ is free with a basis 
$\left\{t_{w} \mid w \in W\right\}$. The multiplication is given by

$$
t_{x} \cdot t_{y}=\sum_{z \in W} \gamma_{x, y, z^{-1}} t_{z} \quad \text { for all } x, y \in W
$$

where the identity element is $1_{J}=\sum_{d \in \mathcal{D}} n_{d} t_{d}$. Furthermore, we have an $A$-algebra homomorphism $\phi: \mathcal{H} \rightarrow A \otimes_{\mathbb{Z}} J$ defined by Lusztig's formula [17, Theorem 18.9]. Then all the methods developped in [17, Chap. 20-24] can be applied to the study of the left cell representations of $\mathcal{H}$.

2.5. Left cell representations. Let $\mathfrak{C}$ be a left cell or, more generally, a union of left cells of $W$. We define an $\mathcal{H}$-module by $[\mathfrak{C}]_{A}:=\mathfrak{I}_{\mathfrak{C}} / \hat{\mathfrak{I}}_{\mathfrak{C}}$, where

$$
\begin{aligned}
& \left.\mathfrak{I}_{\mathfrak{C}}:=\left\langle C_{w}\right| w \leqslant \mathcal{L} z \text { for some } z \in \mathfrak{C}\right\rangle_{A}, \\
& \left.\hat{\mathfrak{I}}_{\mathfrak{C}}:=\left\langle C_{w}\right| w \notin \mathfrak{C}, w \leqslant \mathcal{L} z \text { for some } z \in \mathfrak{C}\right\rangle_{A} .
\end{aligned}
$$

Note that, by the definition of the pre-order relation $\leqslant_{\mathcal{L}}$, these are left ideals in $\mathcal{H}$. Denote by $b_{x}(x \in \mathfrak{C})$ the residue class of $\varepsilon_{x} C_{x}$ in $[\mathfrak{C}]_{A}$. Then the elements $\left\{b_{x} \mid x \in \mathfrak{C}\right\}$ form an $A$-basis of $[\mathfrak{C}]_{A}$ and the action of $C_{w}$ $(w \in W)$ is given by the formula

$$
C_{w} \cdot b_{x}=\varepsilon_{w} \sum_{y \in \mathfrak{C}} h_{w, x, y} b_{y} .
$$

Note that, since $C_{w}^{\dagger}=\varepsilon_{w} C_{w}^{\prime}$, this coincides with the definition in [17, §21.1]. Up to the change of basis $b_{x} \leftrightarrow \varepsilon_{x} b_{x}(x \in \mathfrak{C})$, this also coincides with the original definition by Kazhdan-Lusztig [10.

2.6. Kazhdan-Lusztig's star operation. Let us consider two generators $s \neq t$ in $S$ such that $s t s=t s t$. We set

$$
\mathcal{D}_{R}(s, t):=\{w \in W \mid \text { either } w s<w, w t>w \text { or } w t<w, w s>w\} .
$$

If $w \in \mathcal{D}_{R}(s, t)$, then exactly one of the elements $w s, w t$ belongs to $\mathcal{D}_{R}(s, t)$; we denote it $w^{*}$. The map

$$
\mathcal{D}_{R}(s, t) \rightarrow \mathcal{D}_{R}(s, t), \quad w \mapsto w^{*},
$$

is an involution. It is readily checked that

$$
w \sim_{\mathcal{R}} w^{*} \quad \text { for any } w \in \mathcal{D}_{R}(s, t) .
$$

Now let $w, w_{1} \in W$. Following Kazhdan-Lusztig [10, §5], we write $w \approx w_{1}$ if there exist $s, t \in S$ as above such that $w \in \mathcal{D}_{R}(s, t)$ and $w_{1}=w^{*}$. Now, the relations $\approx$ and $\sim_{\mathcal{L}}$ are compatible, in the following sense. Let $y \in W$ be such that $y \sim_{\mathcal{L}} w$. Then, by [17, 8.6], we also have $y \in \mathcal{D}_{R}(s, t)$. Hence, by [10, Cor. 4.3], we have $y^{*} \sim_{\mathcal{L}} w^{*}$. Consequently, we have a bijection

$$
\mathfrak{C} \stackrel{\sim}{\rightarrow} \mathfrak{C}_{1}, \quad x \mapsto x_{1}:=x^{*},
$$


where $\mathfrak{C}$ is the left cell containing $w$ and $\mathfrak{C}_{1}$ is the left cell containing $w_{1}$. We shall also write $\mathfrak{C} \approx \mathfrak{C}_{1}$ in this situation. By [10, Theorem 4.2] (see also the discussion in [10, §5]), the above bijection has the following property:

$$
h_{s, x, y}=h_{s, x_{1}, y_{1}} \quad \text { for all } s \in S \text { and all } x, y \in \mathfrak{C} .
$$

This means that the action of $C_{s}(s \in S)$ on the $\mathcal{H}$-modules $[\mathfrak{C}]_{A}$ and $\left[\mathfrak{C}_{1}\right]_{A}$ is given by exactly the same formulas with respect to the standard bases of $[\mathfrak{C}]_{A}$ and $\left[\mathfrak{C}_{1}\right]_{A}$, respectively. Since the elements $C_{s}(s \in S)$ generate $\mathcal{H}$ as an $A$-algebra, we can even conclude that

$$
h_{w, x, y}=h_{w, x_{1}, y_{1}} \quad \text { for all } w \in W \text { and all } x, y \in \mathfrak{C} .
$$

We also note the following property of the bijection in (b):

$$
l(x)+l(y) \equiv l\left(x_{1}\right)+l\left(y_{1}\right) \quad \bmod 2, \quad \text { for all } x, y \in \mathfrak{C} .
$$

Indeed, by the definition of $w^{*}$, we have $l\left(w^{*}\right)=l(w) \pm 1$. This immediately yields (d).

2.7. Induction from parabolic subgroups. Let $I \subseteq S$ and consider the parabolic subgroup $W_{I} \subseteq W$. Let $X_{I}$ be the set of distinguished left coset representatives; we have

$$
X_{I}=\left\{w \in W \mid w \text { has minimal length in } w W_{I}\right\} .
$$

Furthermore, the map $X_{I} \times W_{I} \rightarrow W,(x, u) \mapsto x u$, is a bijection and we have $l(x u)=l(x)+l(u)$ for all $u \in W_{I}$ and all $x \in X_{I}$; see [8, §2.1]. Let $\mathcal{H}_{I}=\left\langle T_{w} \mid w \in W_{I}\right\rangle_{A} \subseteq \mathcal{H}$ be the corresponding parabolic subalgebra of $\mathcal{H}$. For any $w \in W_{I}$, we have $C_{w} \in \mathcal{H}_{I}$ and $C_{w}^{\prime} \in \mathcal{H}_{I}$; hence $\left\{C_{w} \mid w \in W_{I}\right\}$ and $\left\{C_{w}^{\prime} \mid w \in W_{I}\right\}$ are the Kazhdan-Lusztig bases of $\mathcal{H}_{I}$.

The linear map $\varepsilon_{I}: \mathcal{H}_{I} \rightarrow A$ defined by $\varepsilon_{I}\left(T_{w}\right)=\varepsilon_{w} v^{-l(w)}$ for any $w \in W_{I}$ is an algebra homomorphism, called the sign representation. We denote by

$$
\operatorname{Ind}_{I}^{S}\left(\varepsilon_{I}\right) \text {, }
$$

the $\mathcal{H}$-module obtained by induction from $\varepsilon_{I}$. According to the situation, we will also use the same symbol for the corresponding character. See 8 , $\S 9.1]$ for the definition and basic properties of induced modules.

Lemma 2.8. Assume that $W_{I}$ is finite and let $w_{I} \in W_{I}$ be the unique element of maximal length. Then the following hold.

(a) For any $w \in W_{I}$, we have $T_{w} C_{w_{I}}=\varepsilon_{w} v^{-l(w)} C_{w_{I}}$.

(b) We have $C_{w_{I}}^{2}=\varepsilon_{w_{I}} v^{-l\left(w_{I}\right)} P_{I} C_{w_{I}}$, where $P_{I}=\sum_{w \in W_{I}} v^{2 l(w)}$.

(c) The set $X_{I} w_{I}$ is a union of left cells in $W$; we have

$$
X_{I} w_{I}=\left\{w \in W \mid w \leqslant{ }_{\mathcal{L}} w_{I}\right\} .
$$

We have $\left[X_{I} w_{I}\right]_{A} \cong \operatorname{Ind}_{I}^{S}\left(\varepsilon_{I}\right) \cong \mathcal{H} C_{w_{I}}$ (isomorphisms as left $\mathcal{H}$ modules). 
Proof. (a) It is well-known that $l\left(w w_{I}\right)=l\left(w_{I}\right)-l(w)$ for all $w \in W_{I}$. Hence, by [17, Cor. 12.2], we obtain

$$
C_{w_{I}}^{\prime}=\sum_{w \in W_{I}} v^{-l\left(w w_{I}\right)} T_{w}=v^{-l\left(w_{I}\right)} \sum_{w \in W_{I}} v^{l(w)} T_{w} .
$$

Applying $j: \mathcal{H} \rightarrow \mathcal{H}$ yields the expression:

$$
C_{w_{I}}=\varepsilon_{w_{I}} v^{l\left(w_{I}\right)} \sum_{w \in W_{I}} \varepsilon_{w} v^{-l(w)} T_{w} .
$$

Now let $s \in I$. Then we have $s w_{I}<w_{I}$ and so the multiplication rule in (2.2) shows that $C_{s} C_{w_{I}}=-\left(v+v^{-1}\right) C_{w_{I}}$. Since $C_{s}=T_{s}-v T_{1}$, this yields $T_{s} C_{w_{I}}=-v^{-1} C_{w_{I}}$. The required formula for $T_{w} C_{w_{I}}$ now follows by a simple induction on $l(w)$.

(b) Using (a), we have

$$
\begin{aligned}
C_{w_{I}}^{2} & =\varepsilon_{w_{I}} v^{l\left(w_{I}\right)} \sum_{w \in W_{I}} \varepsilon_{w} v^{-l(w)} T_{w} C_{w_{I}} \\
& =\varepsilon_{w_{I}} v^{l\left(w_{I}\right)} \sum_{w \in W_{I}} v^{-2 l(w)} C_{w_{I}} \\
& =\varepsilon_{w_{I}} v^{-l\left(w_{I}\right)} \sum_{w \in W_{I}} v^{2\left(l\left(w_{I}\right)-l(w)\right)} C_{w_{I}} \\
& =\varepsilon_{w_{I}} v^{-l\left(w_{I}\right)} P_{I} C_{w_{I}} .
\end{aligned}
$$

(c) Let $w \in W$ be such that $w \leqslant_{\mathcal{L}} w_{I}$. Then the right descent set of $w_{I}$ (which is $I$ ) is contained in the right descent set of $w$; see [17, Lemma 8.6]. Hence we can write $w=x w_{I}$ where $x \in X_{I}$. Conversely, if $x \in X_{I}$ then $l\left(x w_{I}\right)=l(x)+l\left(w_{I}\right)$ and so $x w_{I} \leqslant \mathcal{L} w_{I}$. This yields the equality $X_{I} w_{I}=$ $\left\{w \in W \mid w \leqslant_{\mathcal{L}} w_{I}\right\}$. That equality also shows that $X_{I} w_{I}$ is a union of left cells. Hence the module $\left[X_{I} w_{I}\right]_{A}$ is defined. Now it is known that $C_{x w_{I}}$ is the sum of $T_{x} C_{w_{I}}$ and a linear combination of terms $T_{y} C_{w_{I}}$ where $y \in X_{I}$ and $y<x$. (This is just a special case of [6, Prop. 3.3], for example.) Hence we also have that $T_{x} C_{w_{I}}$ is the sum of $C_{x w_{I}}$ and a linear combination of terms $C_{y w_{I}}$ where $y \in X_{I}$ and $y<x$. Consequently, we have

$$
\left\langle C_{x w_{I}} \mid x \in X_{I}\right\rangle_{A}=\left\langle T_{x} C_{w_{I}} \mid x \in X_{I}\right\rangle_{A},
$$

and it is easily seen that the subspace on the right hand side equals $\mathcal{H} C_{w_{I}}$. Hence, by the definition of induced modules, we have

$$
\left[X_{I} w_{I}\right]_{A} \cong \operatorname{Ind}_{I}^{S}\left(\left\langle C_{w_{I}}\right\rangle_{A}\right) \cong \mathcal{H} C_{w_{I}},
$$

where $\left\langle C_{w_{I}}\right\rangle_{A}$ is an $\mathcal{H}_{I}$-submodule of $\mathcal{H}_{I}$ affording $\varepsilon_{I}$.

\section{Orthogonal REPRESEntations}

We now recall the basic facts concerning the leading matrix coefficients introduced in [5]. For this purpose, we assume from now on that $W$ is a 
finite Coxeter group. We extend scalars from $A$ to the field $K=\mathbb{R}(v)$. Every element $x \in K$ can be written in the form

$$
x=r_{x} v^{\gamma_{x}} f / g \quad \text { where } r_{x} \in \mathbb{R}, \gamma_{x} \in \mathbb{Z} \text { and } f, g \in 1+v \mathbb{R}[v] ;
$$

note that, if $x \neq 0$, then $r_{x}$ and $\gamma_{x}$ indeed are uniquely determined by $x$; for $x=0$, we have $r_{0}=0$ and we set $\gamma_{0}:=+\infty$ by convention. Let

$$
\mathcal{O}:=\left\{x \in K \mid \gamma_{x} \geqslant 0\right\} \quad \text { and } \quad \mathfrak{p}:=\left\{x \in K \mid \gamma_{x}>0\right\} .
$$

Note that $\mathcal{O}$ is nothing but the localisation of $\mathbb{R}[v]$ in the ideal $(v)$; hence, $\mathcal{O}$ is a discrete valuation ring and $\mathfrak{p}$ is the unique maximal ideal of $\mathcal{O}$. The group of units in $\mathcal{O}$ is given by

$$
\mathcal{O}^{\times}=\left\{x \in \mathcal{O} \mid r_{x} \neq 0, \gamma_{x}=0\right\} .
$$

Note that we have

$$
\mathcal{O} \cap \mathbb{R}\left[v, v^{-1}\right]=\mathbb{R}[v] \quad \text { and } \quad \mathfrak{p} \cap \mathbb{R}\left[v, v^{-1}\right]=v \mathbb{R}[v] .
$$

The substitution $v \mapsto 0$ defines an $\mathbb{R}$-linear ring homomorphism $\mathcal{O} \rightarrow \mathbb{R}$ with kernel $\mathfrak{p}$. The image of $x \in \mathcal{O}$ in $\mathbb{R}$ is called the constant term of $x$. Thus, the constant term of $x$ is 0 if $x \in \mathfrak{p}$; the constant term equals $r_{x}$ if $x \in \mathcal{O}^{\times}$.

3.1. Schur elements and $a$-invariants. Extending scalars from $A$ to $K$, we obtain a finite dimensional $K$-algebra $\mathcal{H}_{K}=K \otimes_{A} \mathcal{H}$, with basis $\left\{T_{w} \mid\right.$ $w \in W\}$. It is well-known that $\mathcal{H}_{K}$ is split semisimple and abstractly isomorphic to the group algebra of $W$ over $K$; see, for example, [8, Theorem 9.3.5]. Let $\operatorname{Irr}\left(\mathcal{H}_{K}\right)$ be the set of irreducible characters of $\mathcal{H}_{K}$. We write this set in the form

$$
\operatorname{Irr}\left(\mathcal{H}_{K}\right)=\left\{\chi_{\lambda} \mid \lambda \in \Lambda\right\}
$$

where $\Lambda$ is some finite indexing set. We have a symmetrizing trace $\tau: \mathcal{H}_{K} \rightarrow$ $K$ defined by $\tau\left(T_{1}\right)=1$ and $\tau\left(T_{w}\right)=0$ for $1 \neq w \in W$. We have

$$
\tau\left(T_{w} T_{w^{\prime}}\right)= \begin{cases}1 & \text { if } w^{\prime}=w^{-1} \\ 0 & \text { if } w^{\prime} \neq w^{-1}\end{cases}
$$

see [8, §8.1]. The fact that $\mathcal{H}_{K}$ is split semisimple yields that

$$
\tau=\sum_{\lambda \in \Lambda} \frac{1}{c_{\lambda}} \chi_{\lambda} \quad \text { where } 0 \neq c_{\lambda} \in \mathbb{R}\left[v, v^{-1}\right]
$$

The elements $c_{\lambda}$ are called the Schur elements. By [8, 8.1.8], we have $c_{\lambda}=$ $P_{W} / D_{\lambda}$ where $P_{W}=\sum_{w \in W} v^{2 l(w)}$ is the Poincaré polynomial of $W$ and $D_{\lambda}$ is the "generic degree" associated with $\chi_{\lambda}$. There is a unique $a(\lambda) \in \mathbb{N}$ and a positive real number $r_{\lambda}$ such that

$$
c_{\lambda}=r_{\lambda} v^{-2 a(\lambda)}+\text { combination of higher powers of } v \text {; }
$$

see [13, Chap. 4] and [5, Def. 3.3]. The number $a(\lambda)$ is called the $a$-invariant of $\chi_{\lambda}$. 
3.2. Orthogonal representations. By [5, Prop. 4.3], every $\chi_{\lambda}$ is afforded by a so-called orthogonal representation. This means that there exists a matrix representation $\mathfrak{X}_{\lambda}: \mathcal{H}_{K} \rightarrow M_{d_{\lambda}}(K)$ with character $\chi_{\lambda}$ and an invertible diagonal matrix $P \in M_{d_{\lambda}}(K)$ such that the following conditions hold:

(O1) We have $\mathfrak{X}_{\lambda}\left(T_{w^{-1}}\right)=P^{-1} \cdot \mathfrak{X}_{\lambda}\left(T_{w}\right)^{\text {tr }} \cdot P$ for all $w \in W$, and

(O2) the diagonal entries of $P$ lie in $1+v \mathbb{R}[v]$.

This has the following consequence. Let $\lambda \in \Lambda$ and $1 \leqslant i, j \leqslant d_{\lambda}$. For any $h \in \mathcal{H}_{K}$, we denote by $\mathfrak{X}_{\lambda}^{i j}(h)$ the $(i, j)$-entry of the matrix $\mathfrak{X}_{\lambda}(h)$. Then, by [5. Theorem 4.4 and Remark 4.6], we have

$$
v^{a(\lambda)} \mathfrak{X}_{\lambda}^{i j}\left(T_{w}\right) \in \mathcal{O} \quad \text { and } \quad v^{a(\lambda)} \mathfrak{X}_{\lambda}^{i j}\left(C_{w}\right) \in \mathcal{O}
$$

for any $w \in W$ and

$$
v^{a(\lambda)} \mathfrak{X}_{\lambda}^{i j}\left(T_{w}\right) \equiv v^{a(\lambda)} \mathfrak{X}_{\lambda}^{i j}\left(C_{w}\right) \quad \bmod \mathfrak{p} .
$$

Hence, the above three elements of $\mathcal{O}$ have the same constant term which which we write as $\varepsilon_{w} c_{w, \lambda}^{i j}$. The constants $c_{w, \lambda}^{i j} \in \mathbb{R}$ are called the leading matrix coefficients of $\mathfrak{X}_{\lambda}$. By [5] Theorem 4.4], these coefficients have the following property:

$$
\begin{array}{ll}
c_{w, \lambda}^{i j}=c_{w^{-1}, \lambda}^{j i} & \text { for all } w \in W, \\
c_{w, \lambda}^{i j} \neq 0 & \text { for some } w \in W .
\end{array}
$$

We have the following Schur relations. Let $\lambda, \mu \in \Lambda, 1 \leqslant i, j \leqslant d_{\lambda}$ and $1 \leqslant k, l \leqslant d_{\mu}$; then

$$
\sum_{w \in W} c_{w, \lambda}^{i j} c_{w, \mu}^{k l}=\left\{\begin{array}{cl}
\delta_{i k} \delta_{j l} r_{\lambda} & \text { if } \lambda=\mu \\
0 & \text { if } \lambda \neq \mu
\end{array}\right.
$$

see [5, Theorem 4.4]. (Here $\delta_{i j}$ is the Kronecker delta.) The leading matrix coefficients are related to the left cells of $W$ by the following result. Recall that, given a left cell $\mathfrak{C}$, we have a corresponding left cell module $[\mathfrak{C}]_{A}$. Extending scalars from $A$ to $K$, we obtain an $\mathcal{H}_{K}$-module $[\mathfrak{C}]_{K}$. We denote by $\chi_{\mathfrak{C}}$ the character of $[\mathfrak{C}]_{K}$. Then we have:

Proposition 3.3. Let $\lambda \in \Lambda$ and $\mathfrak{C}$ be a left cell of $W$. Then we have

$$
\left[\chi_{\mathfrak{C}}: \chi_{\lambda}\right] \neq 0 \Leftrightarrow c_{w, \lambda}^{i j} \neq 0 \text { for some } w \in \mathfrak{C} \text { and some } i, j .
$$

Here, $\left[\chi_{\mathfrak{C}}: \chi_{\lambda}\right]$ denotes the multiplicity of $\chi_{\lambda}$ in $\chi_{\mathfrak{C}}$.

Proof. By [5] Prop. 4.7], we have the identity

$$
\sum_{k=1}^{d_{\lambda}} \sum_{y \in \mathfrak{C}}\left(c_{y, \lambda}^{i k}\right)^{2}=\left[\chi_{\mathfrak{C}}: \chi_{\lambda}\right] r_{\lambda}, \quad \text { for any } 1 \leqslant i \leqslant d_{\lambda} .
$$

Now assume first that $\left[\chi_{\mathfrak{C}}: \chi_{\lambda}\right] \neq 0$. Then, clearly, we must have $c_{w, \lambda}^{i j} \neq 0$ for some $w \in \mathfrak{C}$ and some $i, j$. Conversely, assume that $c_{w, \lambda}^{i j} \neq 0$ for some $w \in \mathfrak{C}$ and some $i, j$. Then the term corresponding to $y=w$ and $k=j$ in 
the sum on the left hand side is non-zero. Since there are no cancellations in that sum, the left hand side is non-zero and so $\left[\chi_{\mathfrak{C}}: \chi_{\lambda}\right] \neq 0$, as desired.

The Schur relations lead to particularly strong results when some additional hypotheses are satisfied.

Lemma 3.4. Assume that the following condition is satisfied for all $\lambda \in \Lambda$ :

$$
r_{\lambda}=1 \quad \text { and } \quad c_{w, \lambda}^{i j} \in \mathbb{Z} \quad \text { for all } w \in W \text { and } 1 \leqslant i, j \leqslant d_{\lambda} .
$$

Then the following hold.

(a) We have $c_{w, \lambda}^{i j} \in\{0, \pm 1\}$ for all $w \in W, \lambda \in \Lambda$ and $1 \leqslant i, j \leqslant d_{\lambda}$.

(b) For any $\lambda \in \Lambda$ and $1 \leqslant i, j \leqslant d_{\lambda}$, there exists a unique $w \in W$ such that $c_{w, \lambda}^{i j} \neq 0$; we write $w=\mathbf{w}_{\lambda}(i, j)$. The correspondence $(\lambda, i, j) \mapsto \mathbf{w}_{\lambda}(i, j)$ defines a bijective map

$$
\left.\{(\lambda, i, j)\} \mid \lambda \in \Lambda, 1 \leqslant i, j \leqslant d_{\lambda}\right\} \longrightarrow W .
$$

Proof. This is proved in [7. Lemma 3.8]; see also [5, Theorem 4.10]. In order to illustrate the role of the conditions in $(*)$, we just recall here the proof of the uniqueness statement in (b). Let $\lambda \in \Lambda$ and $1 \leqslant i, j \leqslant d_{\lambda}$. Then consider the Schur relation where $\lambda=\mu, i=l$ and $j=k$. This yields:

$$
\sum_{w \in W}\left(c_{w, \lambda}^{i j}\right)^{2}=r_{\lambda}=1
$$

Since the leading matrix coefficients are integers, we conclude that there exists a unique $w \in W$ such that $c_{w, \lambda}^{i j}= \pm 1$ and $c_{y, \lambda}^{i j}=0$ for all $y \in W \backslash\{w\}$. Thus, we have a map $(\lambda, i, j) \mapsto w=\mathbf{w}_{\lambda}(i, j)$ as in (b). The remaining statements are proved in [loc. cit.].

Remark 3.5. In the setting of Lemma 3.4, let $\lambda \in \Lambda$ and set

$$
\begin{aligned}
\mathfrak{R}(\lambda) & :=\left\{w \in W \mid c_{w, \lambda}^{i j} \neq 0 \text { for some } 1 \leqslant i, j \leqslant d_{\lambda}\right\} \\
& =\left\{\mathbf{w}_{\lambda}(i, j) \mid 1 \leqslant i, j \leqslant d_{\lambda}\right\} .
\end{aligned}
$$

It follows from [5, Theorem 4.4(b)] that

$$
\mathbf{w}_{\lambda}(i, j)^{-1}=\mathbf{w}_{\lambda}(j, i) .
$$

In particular, $\mathbf{w}_{\lambda}(i, j)$ is an involution if and only of $i=j$. Furthermore, let $i, j \in\left\{1, \ldots, d_{\lambda}\right\}$ and define

(b) ${ }^{j} \mathcal{C}_{\lambda}:=\left\{\mathbf{w}_{\lambda}(k, j) \mid 1 \leqslant k \leqslant d_{\lambda}\right\} \quad$ and $\quad \mathcal{C}_{\lambda}^{i}:=\left\{\mathbf{w}_{\lambda}(i, l) \mid 1 \leqslant l \leqslant d_{\lambda}\right\}$.

It is shown in [5, Theorem 4.10] that ${ }^{j} \mathcal{C}_{\lambda}$ is contained in a left cell of $W$ and $\mathcal{C}_{\lambda}^{j}$ is contained in a right cell of $W$. In particular, the whole set $\mathfrak{R}(\lambda)$ is contained in a two-sided cell of $W$.

The following two results will be useful for the identification of irreducible characters; they rely on Proposition 3.3 and Lemma 2.8 . 
Lemma 3.6. Assume that condition (*) in Lemma 3.4 is satisfied. Let $I \subseteq S$ and $\lambda \in \Lambda$ be such that

$$
\left[\operatorname{Ind}_{I}^{S}\left(\varepsilon_{I}\right): \chi_{\lambda}\right] \neq 0
$$

Then the following hold.

(a) We have $w \leqslant_{\mathcal{L R}} w_{I}$ for any $w \in \mathfrak{R}(\lambda)$.

(b) Suppose that $a(\lambda)=l\left(w_{I}\right)$. Then

$$
w_{I} \in \mathfrak{R}(\lambda) \quad \text { and } \quad\left[\chi_{\mathfrak{C}}: \chi_{\lambda}\right] \neq 0,
$$

where $\mathfrak{C}$ is the left cell of $W$ containing $w_{I}$.

Proof. By Lemma 2.8 $X_{I} w_{I}$ is a union of left cells of $W$; furthermore, the left cell module $\left[X_{I} w_{I}\right]_{A}$ affords the character $\operatorname{Ind}_{I}^{S}\left(\varepsilon_{I}\right)$. Hence, our assumption that $\chi_{\lambda}$ occurs with non-zero multiplicity in that induced character implies that there exists some left cell $\mathfrak{C}$ of $W$ such that

$$
\mathfrak{C} \subseteq X_{I} w_{I} \quad \text { and } \quad\left[\chi_{\mathfrak{C}}: \chi_{\lambda}\right] \neq 0 .
$$

By Proposition [3.3, we have $c_{z, \lambda}^{i j} \neq 0$ for some $z \in \mathfrak{C}$ and some $i, j$. In particular, we have $z \in \mathfrak{R}(\lambda)$. Furthermore, writing $z=x w_{I}$ where $x \in X_{I}$, we see that $z \leqslant_{\mathcal{L}} w_{I}$. Since all elements of $\mathfrak{R}(\lambda)$ are contained in a two-sided cell, we obtain (a).

Now suppose that $a(\lambda)=l\left(w_{I}\right)$. Let $e_{I} \in \mathcal{H}_{I, K}$ be the primitive idempotent affording the sign representation $\varepsilon_{I}$ of $\mathcal{H}_{I, K}$. By Frobenius reciprocity, we know that $\varepsilon_{I}$ occurs with non-zero multiplicity in the restriction of $\chi_{\lambda}$ to $\mathcal{H}_{I}$. Hence we conclude that

$$
\chi_{\lambda}\left(e_{I}\right) \in \mathbb{N} \quad \text { and } \quad \chi_{\lambda}\left(e_{I}\right) \neq 0 .
$$

Now, by Lemma 2.8, we have $P_{I} e_{I}=\varepsilon_{w_{I}} v^{l\left(w_{I}\right)} C_{w_{I}}$. Since $P_{I} \in 1+\mathfrak{p}$, we obtain

$$
\begin{aligned}
\sum_{i=1}^{d_{\lambda}} v^{a(\lambda)} & \mathfrak{X}_{\lambda}^{i i}\left(C_{w_{I}}\right) \equiv v^{l\left(w_{I}\right)} \sum_{i=1}^{d_{\lambda}} \mathfrak{X}_{\lambda}^{i i}\left(C_{w_{I}}\right) \\
& \equiv \pm \sum_{i=1}^{d_{\lambda}} \mathfrak{X}_{\lambda}^{i i}\left(e_{I}\right) \equiv \pm \chi_{\lambda}\left(e_{I}\right) \not \equiv 0 \quad \bmod \mathfrak{p} .
\end{aligned}
$$

Hence, there is some $i$ such that $c_{w_{I}, \lambda}^{i i} \neq 0$ and so $w_{I} \in \mathfrak{R}(\lambda)$. Then Proposition $\left[3.3\right.$ shows that $\left[\chi_{\mathfrak{C}}: \chi_{\lambda}\right] \neq 0$, where $\mathfrak{C}$ is the left cell containing $w_{I}$.

Lemma 3.7. Assume that condition (*) in Lemma 3.4 is satisfied. Let $I \subseteq S, x \in X_{I}$ and $\lambda \in \Lambda$ be such that $x w_{I} \in \mathfrak{R}(\lambda)$. Then we have

$$
\left[\operatorname{Ind}_{I}^{S}\left(\varepsilon_{I}\right): \chi_{\lambda}\right] \neq 0 \text {. }
$$

Proof. We have $x w_{\lambda}=\mathbf{w}_{\lambda}(i, j)$ where $1 \leqslant i, j \leqslant d_{\lambda}$. Let $\mathfrak{C}$ be the left cell containing $x w_{\lambda}$. Then Proposition 3.3 shows that $\left[\chi_{\mathfrak{C}}: \chi_{\lambda}\right] \neq 0$. On the other hand, $\chi_{\mathfrak{C}}$ is a summand in $\operatorname{Ind}_{I}^{S}\left(\varepsilon_{I}\right)$, by Lemma 2.8. Consequently, $\chi_{\lambda}$ occurs with non-zero-multiplicity in that induced character, as claimed. 
Example 3.8. Let $W=\mathfrak{S}_{n}$ be the symmetric group on $\{1, \ldots, n\}$. Then $W$ is a Coxeter group with generating set $S=\left\{s_{1}, \ldots, s_{n-1}\right\}$, where $s_{i}=$ $(i, i+1)$ for $1 \leqslant i \leqslant n-1$. The diagram is given as follows.

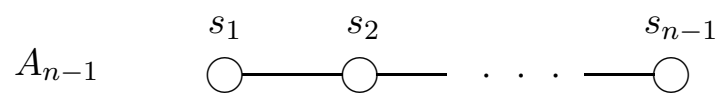

Let $\mathcal{H}=\mathcal{H}_{A}\left(\mathfrak{S}_{n}, S\right)$ be the corresponding Iwahori-Hecke algebra. Let $\Lambda_{n}$ be the set of all partitions of $n$. Then it is well-known (see, for example, Hoefsmit 9] or Dipper-James [2]) that we have a labelling

$$
\operatorname{Irr}\left(\mathcal{H}_{K}\right)=\left\{\chi_{\lambda} \mid \lambda \in \Lambda_{n}\right\}
$$

It will be important to specify precisely the labelling that we are using. A "standard" labelling is defined in [8, §5.4]; however, in order to avoid going back and forth between partitions and their conjugates, we shall change that labelling and simply denote here by $\chi_{\lambda}$ the irreducible character which is labelled by the conjugate partition $\lambda^{*}$ in [loc. cit.]. (See also Corollary 4.5 where we describe a representation affording $\chi_{\lambda}$.) For example, with this convention, $\chi_{\left(1^{n}\right)}$ is the trivial character and $\chi_{(n)}$ is the sign character of $\mathcal{H}_{K}$. The labelling that we have chosen is characterized as follows in terms of induced characters (and this characterisation will be important in Section 4).

Let $\lambda \in \Lambda_{n}$. If $\lambda$ has parts $\lambda_{1} \geqslant \lambda_{2} \geqslant \cdots \geqslant 0$, we set

$$
\lambda_{i}^{+}=\lambda_{1}+\lambda_{2}+\cdots+\lambda_{i} \quad \text { for } i=1,2,3, \ldots
$$

Let $I_{\lambda}:=\{1, \ldots, n\} \backslash\left\{\lambda_{i}^{+} \mid i=1,2, \ldots\right\}$. Then $I_{\lambda} \subseteq S$ and $\mathfrak{S}_{\lambda}:=W_{I_{\lambda}}$ is the Young subgroup corresponding to $\lambda$. Let $\varepsilon_{\lambda}$ be the sign character of the corresponding parabolic subalgebra $\mathcal{H}_{\lambda} \subseteq \mathcal{H}$. Then, by [8, 5.4.7], the labelling of $\operatorname{Irr}\left(\mathcal{H}_{K}\right)$ is uniquely determined by the condition that

$$
\operatorname{Ind}_{I_{\lambda}}^{S}\left(\varepsilon_{\lambda}\right)=\chi_{\lambda}+\text { sum of characters } \chi_{\mu} \text { where } \lambda \triangleleft \mu,
$$

where $\unlhd$ denotes the usual dominance order on $\Lambda_{n}$. (We have $\lambda \unlhd \mu$ if and only if $\lambda_{i}^{+} \leqslant \mu_{i}^{+}$for $i=1,2,3, \ldots$; we write $\lambda \triangleleft \mu$ if $\lambda \unlhd \mu$ and $\lambda \neq \mu$.)

The formula for the Schur elements in [8, Prop. 9.4.5] and the identities in [8, §5.4] now show that we have

$$
r_{\lambda}=1 \quad \text { and } \quad a(\lambda)=l\left(w_{\lambda}\right) \quad \text { for any } \lambda \in \Lambda_{n},
$$

where $w_{\lambda}$ is the unique element of maximal length in $\mathfrak{S}_{\lambda}$. Furthermore, by the discussion in [5, §5], each $\chi_{\lambda}$ is afforded by an orthogonal representation such that the leading matrix coefficients $c_{w, \lambda}^{i j}$ are integers. (Since that discussion is somewhat sketchy, a more rigorous argument is given in [7, Remark 3.7], based on the Dipper-James construction of Hoefsmit's matrices in [3, Theorem 4.9].) Thus, condition (*) in Lemma 3.4 is satisfied. Hence, each element of $\mathfrak{S}_{n}$ lies in $\mathfrak{R}(\lambda)$ for a unique $\lambda \in \Lambda_{n}$. Furthermore, applying Lemma 3.6 and using (a) and (b), we obtain that $w_{\lambda} \in \mathfrak{R}(\lambda)$. Thus, we 
have a partition

$$
\mathfrak{S}_{n}=\coprod_{\lambda \in \Lambda_{n}} \mathfrak{R}(\lambda) \quad \text { where } \quad w_{\lambda} \in \mathfrak{R}(\lambda) .
$$

Remark 3.9. In the setting of Example 3.8, we will henceforth fix, for each $\lambda \in \Lambda_{n}$, an orthogonal representation $\mathfrak{X}_{\lambda}: \mathcal{H}_{K} \rightarrow M_{d_{\lambda}}(K)$ affording $\chi_{\lambda}$ such that the leading matrix coefficients $c_{w, \lambda}^{i j}$ are integers. Thus, the sets $\mathfrak{R}(\lambda)$ are defined and we have $w_{\lambda} \in \mathfrak{R}(\lambda)$. By Remark 3.5. we have

$$
\mathfrak{R}(\lambda)=\left\{\mathbf{w}_{\lambda}(i, j) \mid 1 \leqslant i, j \leqslant d_{\lambda}\right\} .
$$

Since $w_{\lambda} \in \mathfrak{R}(\lambda)$ is an involution, we have $w_{\lambda}=\mathbf{w}_{\lambda}(i, i)$ for some $i$. Conjugating $\mathfrak{X}_{\lambda}$ by a suitable permutation matrix, we may in fact assume that $i=1$. In the sequel, we shall always assume that $\mathfrak{X}_{\lambda}$ has been "normalized" in this way, that is, we have

$$
w_{\lambda}=\mathbf{w}_{\lambda}(1,1) \in \mathfrak{R}(\lambda) .
$$

Let $X_{\lambda}$ be the set of distinguished left coset representatives of $\mathfrak{S}_{\lambda}$ in $\mathfrak{S}_{n}$. Now, by Remark 3.5, the set ${ }^{1} \mathcal{C}_{\lambda}=\left\{\mathbf{w}_{\lambda}(i, 1) \mid 1 \leqslant i \leqslant d_{\lambda}\right\}$ is contained in a left cell of $\mathfrak{S}_{n}$. Hence, by Lemma [2.8, we have

$$
\mathbf{w}_{\lambda}(i, 1)=x_{i} w_{\lambda} \quad \text { where } x_{i} \in X_{\lambda} \text { for } 1 \leqslant i \leqslant d_{\lambda} \text { and } x_{1}=1 .
$$

(An explicit description of the elements $x_{i}$ will be given in Remark [5.7)

We shall need the following result.

Lemma 3.10. Let $\lambda \in \Lambda_{n}$ and $x \in X_{\lambda}$. Assume that $\mu \in \Lambda_{n}$ is such that $x w_{\lambda} \in \mathfrak{R}(\mu)$. Then we have $\lambda \unlhd \mu$; furthermore, if $\mu=\lambda$, then $x=x_{i}$ for some $i \in\left\{1, \ldots, d_{\lambda}\right\}$.

Proof. By Lemma 3.7. $\chi_{\mu}$ occurs with non-zero multiplicity in $\operatorname{Ind}_{I_{\lambda}}^{S}\left(\varepsilon_{\lambda}\right)$. Hence Example 3.8(a) shows that $\lambda \unlhd \mu$. Thus, it remains to consider the case where $\lambda=\mu$. Now, by Lemma 2.8 the set $X_{\lambda} w_{\lambda}$ is a union of left cells of $\mathfrak{S}_{n}$. Let $\mathfrak{C}, \mathfrak{C}^{\prime} \subseteq X_{\lambda} w_{\lambda}$ be left cells such that $w_{\lambda} \in \mathfrak{C}$ and $x w_{\lambda} \in \mathfrak{C}^{\prime \prime}$. First we claim that $\mathfrak{C}=\mathfrak{C}^{\prime}$. This is seen as follows. By Lemma 3.6(b), $\chi_{\lambda}$ occurs with non-zero multiplicity in $\chi_{\mathfrak{C}}$. On the other hand, since $x w_{\lambda} \in \mathfrak{R}(\lambda)$, we can apply Proposition 3.3 to conclude that $\chi_{\lambda}$ also occurs with non-zero multiplicity in $\chi_{\mathfrak{C}^{\prime}}$. Now assume, if possible, that $\mathfrak{C} \neq \mathfrak{C}^{\prime}$. Since $\chi_{\mathfrak{C}}$ and $\chi_{\mathfrak{C}^{\prime}}$ both occur as summands in $\operatorname{Ind}_{I_{\lambda}}^{S}\left(\varepsilon_{\lambda}\right)$ (see Lemma 2.8), we conclude that $\chi_{\lambda}$ occurs with multiplicity at least 2 in that induced character, contradicting Example 3.8(a). Thus, we must have $\mathfrak{C}=\mathfrak{C}^{\prime \prime}$, as claimed.

Now let us write $x w_{\lambda}=\mathbf{w}_{\lambda}(i, j) \in \mathfrak{C}$ where $1 \leqslant i, j \leqslant d_{\lambda}$. As in the proof of Proposition 3.3, we have the identity

$$
\sum_{k=1}^{d_{\lambda}} \sum_{y \in \mathfrak{C}}\left(c_{y, \lambda}^{i k}\right)^{2}=\left[\chi_{\mathfrak{C}}: \chi_{\lambda}\right] r_{\lambda}=1 .
$$

As discussed in Remark 3.9] we have $x_{i} w_{\lambda}=\mathbf{w}_{\lambda}(i, 1) \in \mathfrak{C}$. Hence this term gives a non-zero contribution to the sum on the left hand side. On the other 
hand, we also have $c_{x w_{\lambda}, \lambda}^{i j} \neq 0$ and this gives a non-zero contribution. Since the right hand side equals 1 , we conclude that $x w_{\lambda}=x_{i} w_{\lambda}$ and so $x=x_{i}$, as desired.

Remark 3.11. Let $\lambda, \mu \in \Lambda_{n}$ be such that $\lambda \unlhd \mu$. Then the multiplicity

$$
\left[\operatorname{Ind}_{I_{\lambda}}^{S}\left(\varepsilon_{\lambda}\right): \chi_{\mu}\right]
$$

can be expressed in a purely combinatorial way, by Young's rule. In particular, it is known that the above multiplicity is non-zero; see, for example, Murphy [21, Theorem 7.2]. So Lemma 3.6 shows that $w \leqslant_{\mathcal{L R}} w_{\lambda}$ for any $w \in \mathfrak{R}(\mu)$. Using Example 3.8(c), we conclude that

$$
\lambda \unlhd \mu \quad \Rightarrow \quad w_{\mu} \leqslant \mathcal{L R} w_{\lambda} .
$$

In Corollary 5.1, we will see that the converse also holds, but the proof requires much more work.

\section{The Murphy BASIS}

Throughout this and the following section, we consider the Iwahori-Hecke algebra $\mathcal{H}=\mathcal{H}_{A}\left(\mathfrak{S}_{n}, S\right)$, as in Example 3.8 The conventions in Remark 3.9 will also remain in force.

In two fundamental articles [20] and 21], Murphy has constructed a new basis $\left\{x_{\mathfrak{s t}}\right\}$ of $\mathcal{H}$ whose elements are indexed by pairs of standard $\lambda$-tableaux, for various partitions $\lambda$ of $n$. As we shall see, the elements $x_{\mathfrak{s t}}$ are defined as a mixture of basis elements $T_{w}$ and $C_{w}$. Our aim is to establish a direct link with the Kazhdan-Lusztig basis; this will be achieved in Theorem 4.10.

We begin by recalling some purely combinatorial notions, where we follow 21] (but we let $\mathfrak{S}_{n}$ act on the left on $\{1, \ldots, n\}$ ). Another reference is the exposition by Mathas 18 .

As before, let $\Lambda_{n}$ be the set of all partitions of $n$. Let $\lambda \in \Lambda_{n}$ and let $\lambda_{1} \geqslant \ldots \geqslant \lambda_{r}>0$ be the non-zero parts of $\lambda$. The correspdonding Young diagram $[\lambda]$ is the set of all pairs $(i, j)$ such that $1 \leqslant i \leqslant r$ and $1 \leqslant j \leqslant \lambda_{i}$. A $\lambda$-tableau is a bijection $\mathfrak{t}:[\lambda] \rightarrow\{1, \ldots, n\}$. We say that $\mathfrak{t}$ is row-standard if the sequence $\mathfrak{t}(i, 1), \mathfrak{t}(i, 2), \ldots$ is strictly increasing for each $i$; similarly, we say that $\mathfrak{t}$ is column-standard if the sequence $\mathfrak{t}(1, j), \mathfrak{t}(2, j), \ldots$ is strictly increasing for each $j$. We say that $\mathfrak{t}$ is standard if $\mathfrak{t}$ is row-standard and column-standard. We denote by $\mathfrak{t}^{\lambda}$ the unique standard $\lambda$-tableau such that

$$
\mathfrak{t}^{\lambda}(i, j)=\lambda_{1}+\lambda_{2}+\cdots+\lambda_{i-1}+j \quad \text { for } 1 \leqslant i \leqslant r \text { and } 1 \leqslant j \leqslant \lambda_{i} .
$$

Here are some examples, where $n=5$ and $\lambda=(3,2)$ :

$$
\mathfrak{t}^{\lambda}=\begin{array}{|l|l|l|}
\hline 1 & 2 & 3 \\
\hline 4 & 5 &
\end{array}
$$

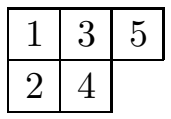

(standard)

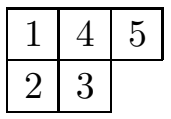

(row-standard)

The group $\mathfrak{S}_{n}$ acts naturally on $\lambda$-tableaux, the action being given by $(w \cdot \mathfrak{t})(i, j):=w \cdot \mathfrak{t}(i, j)$ for a tableau $\mathfrak{t}$ and $w \in \mathfrak{S}_{n}$. The row stabiliser of 
$\mathfrak{t}^{\lambda}$ is the Young subgroup $\mathfrak{S}_{\lambda}$. As in Remark 3.9, let $X_{\lambda}$ be the set of distinguished left coset representatives of $\mathfrak{S}_{\lambda}$ in $\mathfrak{S}_{n}$. By Dipper-James [2, Lemma 1.1], we have the following explicit description:

$$
X_{\lambda}=\left\{w \in \mathfrak{S}_{n} \mid w \cdot \mathbf{t}^{\lambda} \text { is row-standard }\right\} .
$$

As in [loc. cit.], if $\mathfrak{t}$ is a row-standard $\lambda$-tableau, the unique element $d \in X_{\lambda}$ such that $\mathfrak{t}=d \cdot \mathfrak{t}^{\lambda}$ will be denoted by $d(\mathfrak{t})$.

Let $\mathbb{T}(\lambda)$ be the set of standard $\lambda$-tableaux.

Theorem 4.1 (Murphy 20], 21]). For any $\lambda \in \Lambda_{n}$ and $\mathfrak{s}, \mathfrak{t} \in \mathbb{T}(\lambda)$, we define elements of $\mathcal{H}$ by

$$
x_{\lambda}:=\sum_{w \in \mathfrak{S}_{\lambda}} v^{l(w)} T_{w} \quad \text { and } \quad x_{\mathfrak{s t}}:=T_{d(\mathfrak{s})} x_{\lambda} T_{d(\mathfrak{t})}-1
$$

Then the following hold.

(a) The set $\left\{x_{\mathfrak{s t}} \mid \mathfrak{s}, \mathfrak{t} \in \mathbb{T}(\lambda)\right.$ for some $\left.\lambda \in \Lambda_{n}\right\}$ is an $A$-basis of $\mathcal{H}$.

(b) For any $\lambda \in \Lambda_{n}$, let $N^{\lambda}$ be the $A$-submodule of $\mathcal{H}$ spanned by all elements $x_{\mathfrak{s t}}$ where $\mathfrak{s}, \mathfrak{t} \in \mathbb{T}(\mu)$ for some $\mu \in \Lambda$ such that $\lambda \unlhd \mu$. Then $N^{\lambda}$ is a two-sided ideal in $\mathcal{H}$.

The statement in (a) can be found in [20, Theorem 3.9] or, with a somewhat different proof, in [21, Theorem 4.17]. The statement in (b) is proved in [21, Theorem 4.18] (see also [21, §5]). Note that the element that we denote by $T_{w}$ corresponds to the element $v^{-l(w)} T_{w}$ in Murphy's notation. Thus, the element denoted by $x_{\lambda}$ in the above statement is exactly the same as in Murphy's work; the element denoted by $x_{\mathfrak{s t}}$ is only the same up to a power of $v$. However, this does not affect the validity of (a) and (b) since $v$ is invertible in $A$.

Murphy also obtains the following result concerning the Specht modules of $\mathcal{H}$. For any $\lambda \in \Lambda_{n}$, let $\hat{N}^{\lambda}$ be the $A$-submodule of $\mathcal{H}$ spanned by all $x_{\mathfrak{s t}}$ where $\mathfrak{s}, \mathfrak{t} \in \mathbb{T}(\mu)$ for some $\mu \in \Lambda$ such that $\lambda \triangleleft \mu$, that is, $\lambda \unlhd \mu$ and $\lambda \neq \mu$. Thus, we have

$$
\hat{N}^{\lambda}=\sum_{\mu} N^{\mu}
$$

where the sum runs over all $\mu \in \Lambda_{n}$ such that $\lambda \triangleleft \mu$. In particular, $\hat{N}^{\lambda}$ is a two-sided ideal and we have $N^{\lambda}=\mathcal{H} x_{\lambda} \mathcal{H}+\hat{N}^{\lambda}$.

Theorem 4.2 (Murphy [21, §5]). Let $\lambda \in \Lambda_{n}$. Then

$$
\tilde{\mathcal{S}}^{\lambda}:=\left\langle T_{d(\mathfrak{s})} x_{\lambda}+\hat{N}^{\lambda} \mid \mathfrak{s} \in \mathbb{T}(\lambda)\right\rangle_{A} \subseteq N^{\lambda} / \hat{N}^{\lambda}
$$

is a left $\mathcal{H}$-module, and $N^{\lambda} / \hat{N}^{\lambda}$ is a direct sum of $|\mathbb{T}(\lambda)|$ copies of $\tilde{\mathcal{S}}^{\lambda}$. Furthermore, the $\mathcal{H}_{K}$-module $K \otimes_{A} \tilde{\mathcal{S}}^{\lambda}$ is simple, and $\tilde{\mathcal{S}}^{\lambda}$ is the contragredient dual of the Dipper-James Specht module defined in [2].

The following reformulation of Theorem 4.1 will eventually allow us to establish a direct connection between the Murphy basis and the KazhdanLusztig basis. 
Corollary 4.3. For any $\lambda \in \Lambda_{n}$ and $\mathfrak{s}, \mathfrak{t} \in \mathbb{T}(\lambda)$, we set

$$
\tilde{y}_{\mathfrak{s t}}:=T_{d(\mathfrak{s})} C_{w_{\lambda}} T_{d(\mathfrak{t})^{-1}} \in \mathcal{H} .
$$

Then $\tilde{y}_{\mathfrak{s t}}= \pm v^{l\left(w_{\lambda}\right)} j\left(x_{\mathfrak{s t}}\right)$ for all $\mathfrak{s}, \mathfrak{t}$. Consequently, the following hold.

(a) The set $\left\{\tilde{y}_{\mathfrak{s t}} \mid \mathfrak{s}, \mathfrak{t} \in \mathbb{T}(\lambda)\right.$ for some $\left.\lambda \in \Lambda_{n}\right\}$ is an $A$-basis of $\mathcal{H}$.

(b) For any $\lambda \in \Lambda_{n}$, let $\mathcal{N}^{\lambda}$ be the $A$-submodule of $\mathcal{H}$ spanned by all elements $\tilde{y}_{\mathfrak{s t}}$ where $\mathfrak{s}, \mathfrak{t} \in \mathbb{T}(\mu)$ for some $\mu \in \Lambda$ such that $\lambda \unlhd \mu$. Then $\mathcal{N}^{\lambda}$ is a two-sided ideal in $\mathcal{H}$.

Proof. Using the expression for $C_{w_{\lambda}}^{\prime}$ in the proof of Lemma 2.8, we immediately see that $x_{\lambda}=v^{l\left(w_{\lambda}\right)} C_{w_{\lambda}}^{\prime}$. Next recall that, for any $w \in \mathfrak{S}_{n}$, we have $j\left(T_{w}\right)=\varepsilon_{w} T_{w}$ and $j\left(C_{w}^{\prime}\right)=\varepsilon_{w} C_{w}$. Hence we obtain

$$
\begin{aligned}
j\left(x_{\mathfrak{s t}}\right) & =j\left(T_{d(\mathfrak{s})}\right) j\left(x_{\lambda}\right) j\left(T_{d(\mathfrak{t})^{-1}}\right) \\
& =\varepsilon_{d(\mathfrak{s})} \varepsilon_{d(\mathfrak{t})} \varepsilon_{w_{\lambda}} v^{-l\left(w_{\lambda}\right)} T_{d(\mathfrak{s})} C_{w_{\lambda}} T_{d(\mathfrak{t})} \\
& = \pm v^{-l\left(w_{\lambda}\right)} \tilde{y}_{\mathfrak{s t} .}
\end{aligned}
$$

Since $j: \mathcal{H} \rightarrow \mathcal{H}$ is a ring involution, we now see that the statements in Theorem 4.1 hold with $x_{\mathfrak{s t}}$ replaced by $\tilde{y}_{\mathfrak{s t}}$ throughout.

Remark 4.4. For any $\lambda \in \Lambda_{n}$, let $\hat{\mathcal{N}}^{\lambda}$ be the $A$-submodule of $\mathcal{H}$ spanned by all elements $\tilde{y}_{\mathfrak{s t}}$ where $\mathfrak{s}, \mathfrak{t} \in \mathbb{T}(\mu)$ for some $\mu \in \Lambda$ such that $\lambda \triangleleft \mu$. Thus, we have

$$
\hat{\mathcal{N}}^{\lambda}=\sum_{\mu} \mathcal{N}^{\mu}
$$

where the sum runs over all $\mu \in \Lambda_{n}$ such that $\lambda \triangleleft \mu$. In particular, $\hat{\mathcal{N}}^{\lambda}$ is a two-sided ideal and we have $\mathcal{N}^{\lambda}=\mathcal{H} C_{w_{\lambda}} \mathcal{H}+\hat{\mathcal{N}}^{\lambda}$. We claim that

$$
j\left(N^{\lambda}\right)=\left(N^{\lambda}\right)^{\dagger}=\mathcal{N}^{\lambda} \quad \text { and } \quad j\left(\hat{N}^{\lambda}\right)=\left(\hat{N}^{\lambda}\right)^{\dagger}=\hat{\mathcal{N}}^{\lambda} .
$$

Indeed, in the proof of Corollary 4.3, we have seen that $j\left(x_{\lambda}\right)= \pm v^{-l\left(w_{\lambda}\right)} C_{w_{\lambda}}$. But then we also have

$$
x_{\lambda}^{\dagger}=\overline{j\left(x_{\lambda}\right)}= \pm v^{l\left(w_{\lambda}\right)} \bar{C}_{w_{\lambda}}= \pm v^{l\left(w_{\lambda}\right)} C_{w_{\lambda}} .
$$

An easy induction on the dominance order, using the relations

$$
N^{\lambda}=\mathcal{H} x_{\lambda} \mathcal{H}+\hat{N}^{\lambda} \quad \text { and } \quad \mathcal{N}^{\lambda}=\mathcal{H} C_{w_{\lambda}} \mathcal{H}+\hat{\mathcal{N}}^{\lambda},
$$

now shows that $\left(N^{\lambda}\right)^{\dagger}=\mathcal{N}^{\lambda}=j\left(N^{\lambda}\right)$. Since this holds for all $\lambda \in \Lambda_{n}$, we also have $\left(\hat{N}^{\lambda}\right)^{\dagger}=\hat{\mathcal{N}}^{\lambda}=j\left(\hat{N}^{\lambda}\right)$. Thus, the above claim is proved.

The identification of $\chi_{\lambda}$ in the following result relies on our conventions for labelling the irreducible characters in Example 3.8.

Corollary 4.5. Let $\lambda \in \Lambda_{n}$. Then

$$
\mathcal{E}^{\lambda}:=\left\langle T_{d(\mathfrak{s})} C_{w_{\lambda}}+\hat{\mathcal{N}}^{\lambda} \mid \mathfrak{s} \in \mathbb{T}(\lambda)\right\rangle_{A} \subseteq \mathcal{N}^{\lambda} / \hat{\mathcal{N}}^{\lambda}
$$

is a left $\mathcal{H}$-module, and $\mathcal{N}^{\lambda} / \hat{\mathcal{N}}^{\lambda}$ is a direct sum of $|\mathbb{T}(\lambda)|$ copies of $\mathcal{E}^{\lambda}$. The character afforded by $K \otimes_{A} \mathcal{E}^{\lambda}$ is $\chi_{\lambda}$, and we have $d_{\lambda}=|\mathbb{T}(\lambda)|$. 
Proof. By Remark 4.4, the ring involution $j: \mathcal{H} \rightarrow \mathcal{H}$ transforms $N^{\lambda}$ and $\hat{N}^{\lambda}$ into $\mathcal{N}^{\lambda}$ and $\hat{\mathcal{N}}^{\lambda}$, respectively. Hence we have an induced isomorphism of additive groups

$$
\theta: N^{\lambda} / \hat{N}^{\lambda} \rightarrow \mathcal{N}^{\lambda} / \hat{\mathcal{N}}^{\lambda}
$$

such that $\theta(h . e)=j(h) . \theta(e)$ for all $h \in \mathcal{H}$ and $e \in N^{\lambda} / \hat{N}^{\lambda}$. Now, by Corollary 4.3. we have $j\left(x_{\lambda}\right)= \pm v^{-l\left(w_{\lambda}\right)} C_{w_{\lambda}}$ and so $\theta\left(\tilde{\mathcal{S}}^{\lambda}\right)=\mathcal{E}^{\lambda}$. Hence Theorem 4.2 implies that $\mathcal{E}^{\lambda}$ is a left $\mathcal{H}$-module such that $K \otimes_{A} \mathcal{E}^{\lambda}$ is simple; furthermore, $\mathcal{N}^{\lambda} / \hat{\mathcal{N}}^{\lambda}$ is a direct sum of $|\mathbb{T}(\lambda)|$ copies of $\mathcal{E}^{\lambda}$.

Thus, we see that it will be sufficient to prove that the character afforded by $\left.\left(\mathcal{N}^{\lambda}\right) / \hat{\mathcal{N}}^{\lambda}\right)_{K}$ is $d_{\lambda} \chi_{\lambda}$. (The subscript $K$ indicates extension of scalars from $A$ to $K$.) For this purpose, we proceed by downward induction on the dominance order. The unique maximal element for that order is the partition $\lambda=(n)$. In this case, we have $\mathfrak{S}_{(n)}=\mathfrak{S}_{n}$ and $X_{(n)}=\{1\}$. Hence we have $\mathcal{N}^{(n)}=\left\langle C_{w_{(n)}}\right\rangle_{A}$ and $\hat{\mathcal{N}}^{(n)}=\{0\}$. By Lemma 2.8. $\mathcal{N}^{(n)}$ affords the sign character. On the other hand, by Example 3.8 $\chi_{(n)}$ also is the sign character and $d_{(n)}=1$. Thus, the assertion holds in this case. Now let $\lambda \neq(n)$. First we prove the following two statements.

(1) If $\lambda \triangleleft \mu$, then $\chi_{\mu}$ does not occur in the character of $\left(\mathcal{N}^{\lambda} / \hat{\mathcal{N}}^{\lambda}\right)_{K}$.

(2) Consider the left $\mathcal{H}$-module $M^{\lambda}:=\mathcal{H} C_{w_{\lambda}}$. Then $\left(\mathcal{N}^{\lambda} / \hat{\mathcal{N}}^{\lambda}\right)_{K}$ and $M_{K}^{\lambda}$ have a common simple constituent.

To prove (1), we argue as follows. As a left $\mathcal{H}_{K}$-module, we have

$$
\mathcal{N}_{K}^{\lambda} \cong\left(\mathcal{N}^{\lambda} / \hat{\mathcal{N}}^{\lambda}\right)_{K} \oplus \hat{\mathcal{N}}_{K}^{\lambda} \quad \text { where } \quad \mathcal{N}_{K}^{\mu} \subseteq \hat{\mathcal{N}}_{K}^{\lambda} .
$$

By induction, we already know that $d_{\mu} \chi_{\mu}$ is the character afforded by $\left(\mathcal{N}^{\mu} / \hat{\mathcal{N}}^{\mu}\right)_{K}$. Hence, the character of $\hat{\mathcal{N}}^{\lambda}$ contains $d_{\mu} \chi_{\mu}$ as a summand. Since $d_{\mu}$ is the multiplicity of $\chi_{\mu}$ in the character of the regular representation of $\mathcal{H}_{K}$ and $\mathcal{N}_{K}^{\lambda}$ is contained in $\mathcal{H}_{K}$, we conclude that (1) holds. Now let us prove (2). Since $C_{w_{\lambda}} \in \mathcal{N}^{\lambda}$ and $C_{w_{\lambda}} \notin \hat{\mathcal{N}}^{\lambda}$, the inclusion $M^{\lambda} \subseteq \mathcal{N}^{\lambda}$ induces a non-zero homomorphism of $\mathcal{H}$-modules $\varphi: M^{\lambda} \rightarrow \mathcal{N}^{\lambda} / \hat{\mathcal{N}}^{\lambda}$. Hence, (2) follows.

Now we can determine the character afforded by $\left(\mathcal{N}^{\lambda} / \hat{\mathcal{N}}^{\lambda}\right)_{K}$. Let $\mu \in \Lambda_{n}$ be such that $\chi_{\mu}$ is the character of a common simple component as in (2). By Lemma 2.8, we have $M^{\lambda} \cong \operatorname{Ind}_{I_{\lambda}}^{S}\left(\varepsilon_{\lambda}\right)$. Hence, since $\chi_{\mu}$ occurs in $M_{K}^{\lambda}$, we must have $\lambda \unlhd \mu$ by Example [3.8(a). But then (1) yields that $\lambda=\mu$. Thus, $\chi_{\lambda}$ occurs in the character of $\left(\mathcal{N}^{\lambda} / \hat{\mathcal{N}}^{\lambda}\right)_{K}$. Since the latter module is a direct sum of $|\mathbb{T}(\lambda)|$ copies of $\mathcal{E}_{K}^{\lambda}$, we conclude that $\chi_{\lambda}$ is the character of $\mathcal{E}_{K}^{\lambda}$. Since $\mathcal{E}_{K}^{\lambda}$ has dimension $|\mathbb{T}(\lambda)|$, this also yields that $|\mathbb{T}(\lambda)|=d_{\lambda}$, as required.

Corollary 4.6. Let $\lambda, \mu \in \Lambda_{n}$ and $h \in \mathcal{N}^{\lambda}$. Then $\mathfrak{X}_{\mu}(h)=0$ unless $\lambda \unlhd \mu$.

Proof. Let us assume that $\mathfrak{X}_{\mu}(h) \neq 0$. We must show that $\lambda \unlhd \mu$. To see this, consider the representation afforded by the left $\mathcal{H}$-module $\mathcal{N}^{\mu} / \hat{\mathcal{N}}^{\mu}$. Extending scalars to $K$ and using Corollary 4.5, we see that this representation 
is equivalent to a direct sum of $d_{\mu}$ copies of $\mathfrak{X}_{\mu}$. So the condition $\mathfrak{X}_{\mu}(h) \neq 0$ implies that $h .\left(\mathcal{N}^{\mu} / \hat{\mathcal{N}}^{\mu}\right) \neq 0$. This means that there exist some standard $\mu$-tableaux $\mathfrak{s}, \mathfrak{s}_{1}, \mathfrak{t}, \mathfrak{t}_{1}$ such that $\tilde{y}_{\mathfrak{s}_{1} \mathfrak{t}_{1}}$ occurs with non-zero coefficient in the decomposition of $h \tilde{y}_{\mathfrak{s t}}$. Now, since $h \in \mathcal{N}^{\lambda}$ and $\mathcal{N}^{\lambda}$ is a two-sided ideal, we can write $h \tilde{y}_{\mathfrak{s t}}$ as a linear combination of terms $\tilde{y}_{\mathfrak{u v}}$ where $\mathfrak{u}, \mathfrak{v}$ are standard $\nu$-tableaux for partitions $\nu \in \Lambda_{n}$ such that $\lambda \unlhd \nu$. One of the terms in that linear combination is $\tilde{y}_{\mathfrak{s}_{1} \mathfrak{t}_{1}}$. Hence, we conclude that $\lambda \unlhd \mu$, as claimed.

The following definition relies on our conventions in Remark 3.9 concerning the labelling of the elements in the sets $\mathfrak{R}(\lambda)$.

Definition 4.7. Let $w \in \mathfrak{S}_{n}$. Let $\lambda \in \Lambda_{n}$ be such that $w \in \mathfrak{R}(\lambda)$. Then $w=\mathbf{w}_{\lambda}(i, j)$ where $1 \leqslant i, j \leqslant d_{\lambda}$ are uniquely determined. We set

$$
\lambda_{w}:=\lambda, \quad P_{\lambda}:=P_{I_{\lambda}}, \quad \alpha_{w}:=a(\lambda)=l\left(w_{\lambda}\right)
$$

(see Example 3.8) and

$$
Z_{w}:=\frac{1}{P_{\lambda}} \varepsilon_{w_{\lambda}} v^{l\left(w_{\lambda}\right)} C_{x_{i} w_{\lambda}} C_{w_{\lambda} x_{j}^{-1}} \in \mathcal{H}_{K}
$$

Note that we do need to extend scalars from $A$ to $K$ in order to define the above element.

The following result shows that $Z_{w}$ actually lies in $\mathcal{H}$.

Lemma 4.8. We have $Z_{w} \in \mathcal{N}^{\lambda} \subseteq \mathcal{H}$ and $\bar{Z}_{w}=Z_{w}$. For $w=w_{\lambda}$, we have $Z_{w_{\lambda}}=C_{w_{\lambda}}$.

Proof. By [6. Prop. 3.3], we have

$$
\begin{aligned}
C_{x_{i} w_{\lambda}} & =A \text {-linear combination of terms } T_{x} C_{w_{\lambda}} \text { where } x \in X_{\lambda}, \\
C_{w_{\lambda} x_{j}^{-1}} & =A \text {-linear combination of terms } C_{w_{\lambda}} T_{y^{-1}} \text { where } y \in X_{\lambda} .
\end{aligned}
$$

(We have already used this fact in the proof of Lemma 2.8.) Hence, $Z_{w}$ is an $A$-linear combination of terms of the form

$$
\frac{1}{P_{\lambda}} \varepsilon_{w_{\lambda}} v^{l\left(w_{\lambda}\right)} T_{x} C_{w_{\lambda}}^{2} T_{y^{-1}} \quad \text { where } x, y \in X_{\lambda} .
$$

Using Lemma 2.8 each of the above terms is equal to $T_{x} C_{w_{\lambda}} T_{y^{-1}}$ and, hence, lies in $\mathcal{H}$. Consequently, we also have that $Z_{w}$ lies in $\mathcal{H} C_{w_{\lambda}} \mathcal{H}$, and this is contained in $\mathcal{N}^{\lambda}$ by Remark 4.4. Finally, note that $v^{l\left(w_{\lambda}\right)} \bar{P}_{\lambda}=v^{-l\left(w_{\lambda}\right)} P_{\lambda}$. This yields $\bar{Z}_{w}=Z_{w}$.

Lemma 4.9. Let $w \in \mathfrak{R}(\lambda)$ and $1 \leqslant k, l \leqslant d_{\lambda}$. Then we have

$$
v^{a(\lambda)} \mathfrak{X}_{\lambda}^{k l}\left(Z_{w}\right) \in \mathcal{O} \quad \text { and } \quad v^{a(\lambda)} \mathfrak{X}_{\lambda}^{k l}\left(Z_{w}\right) \equiv \pm \delta_{i k} \delta_{j l} \quad \bmod \mathfrak{p},
$$

where $i, j \in\left\{1, \ldots, d_{\lambda}\right\}$ are such that $w=\mathbf{w}_{\lambda}(i, j)$. 
Proof. We write $w=\mathbf{w}_{\lambda}(i, j)$ where $1 \leqslant i, j \leqslant d_{\lambda}$. We have $a(\lambda)=l\left(w_{\lambda}\right)$ by Example 3.8. Hence we obtain

$$
\begin{aligned}
v^{a(\lambda)} \mathfrak{X}_{\lambda}^{k l}\left(Z_{w}\right) & = \pm \frac{1}{P_{\lambda}} v^{2 a(\lambda)} \mathfrak{X}_{\lambda}^{k l}\left(C_{x_{i} w_{\lambda}} C_{w_{\lambda} x_{j}^{-1}}\right) \\
& = \pm \frac{1}{P_{\lambda}} \sum_{r=1}^{d_{\lambda}}\left(v^{a(\lambda)} \mathfrak{X}_{\lambda}^{k r}\left(C_{x_{i} w_{\lambda}}\right)\right)\left(v^{a(\lambda)} \mathfrak{X}_{\lambda}^{r l}\left(C_{w_{\lambda} x_{j}^{-1}}\right)\right) .
\end{aligned}
$$

First of all, this shows that the above expression lies in $\mathcal{O}$; note that $P_{\lambda} \in 1+v \mathbb{Z}[v]$. Furthermore, its constant term can be expressed by the leading matrix coefficients of $x_{i} w_{\lambda}$ and $x_{j} w_{\lambda}$. Indeed, since $x_{i} w_{\lambda}=\mathbf{w}_{\lambda}(i, 1)$, Lemma 3.4 shows that

$$
v^{a(\lambda)} \mathfrak{X}_{\lambda}^{k r}\left(C_{x_{i} w_{\lambda}}\right) \equiv \varepsilon_{x_{i} w_{\lambda}} c_{x_{i} w_{\lambda}, \lambda}^{k r} \equiv \pm \delta_{k i} \delta_{r 1} \quad \bmod \mathfrak{p} .
$$

Similarly, since $w_{\lambda} x_{j}^{-1}=\mathbf{w}_{\lambda}(1, j)$, we have

$$
v^{a(\lambda)} \mathfrak{X}_{\lambda}^{r l}\left(C_{w_{\lambda} x_{j}^{-1}}\right) \equiv \pm \delta_{l j} \delta_{r 1} \quad \bmod \mathfrak{p} .
$$

Now note that $P_{\lambda} \in 1+\mathfrak{p}$ and so $P_{\lambda}^{-1} \equiv 1 \bmod \mathfrak{p}$. Hence, we obtain

$$
v^{a(\lambda)} \mathfrak{X}_{\lambda}^{k l}\left(Z_{w}\right) \equiv \pm \delta_{k i} \delta_{l j} \quad \bmod \mathfrak{p}
$$

as desired.

Theorem 4.10. Let $\lambda \in \Lambda_{n}$. Then the following hold.

(a) $\quad \eta_{w} C_{w} \in Z_{w}+\hat{\mathcal{N}}^{\lambda} \subseteq \mathcal{N}^{\lambda} \quad$ for all $w \in \mathfrak{R}(\lambda)$, where $\eta_{w}= \pm 1$,

(b) $\quad \mathcal{N}^{\lambda}=\left\langle C_{w}\right| w \in \mathfrak{R}(\mu)$ for some $\mu \in \Lambda_{n}$ such that $\left.\lambda \unlhd \mu\right\rangle_{A}$.

(The sign in (a) will be determined explicitly in Corollary [5.11,)

Proof. (a) We proceed by downward induction on the dominance order. The unique maximal element for that order is the partition $\lambda=(n)$. In this case, we have $\mathfrak{S}_{(n)}=\mathfrak{S}_{n}, X_{(n)}=\{1\}$ and $d_{(n)}=1$. Consequently, we have

$$
\mathfrak{R}((n))=\left\{w_{(n)}\right\} \quad \text { and } \quad \mathcal{N}^{(n)}=\left\langle C_{w_{(n)}}\right\rangle_{A} .
$$

On the other hand, we also have $Z_{w_{(n)}}=C_{w_{(n)}}$. Hence the desired statement holds in this case. Now consider an arbitrary partition $\lambda \neq(n)$ and assume that the desired statements hold for all elements in sets $\mathfrak{R}(\nu)$ where $\lambda \triangleleft \nu$. Let $1 \leqslant i, j \leqslant d_{\lambda}$ be such that $w=\mathbf{w}_{\lambda}(i, j)$. By Lemma 4.8, we can write

$$
Z_{w}=\sum_{z \in \mathfrak{S}_{n}} f_{z} C_{z} \quad \text { where } f_{z} \in A
$$

Claim (1). We have $f_{z} \in \mathbb{Z}$ for all $z \in \mathfrak{S}_{n}$ such that $\lambda \Varangle \lambda_{z}$.

This is seen as follows. Let $\mathcal{A}$ be the collection of all $z \in \mathfrak{S}_{n}$ such that $f_{z} \neq 0$ and $\lambda \Varangle \lambda_{z}$. If $\mathcal{A}=\varnothing$, there is nothing to be proved. Now assume 
that $\mathcal{A} \neq \varnothing$. Since $Z_{w}=\bar{Z}_{w}$, we have $f_{z}=\bar{f}_{z}$ for all $z \in \mathfrak{S}_{n}$. Hence it will be sufficient to show that the non-negative number

$$
m:=\min \left\{i \geqslant 0 \mid v^{i} f_{z} \in \mathbb{Z}[v] \text { for all } z \in \mathcal{A}\right\}
$$

is actually equal to 0 . Let $z^{\prime} \in \mathcal{A}$ be such that $v^{m} f_{z^{\prime}} \in \mathbb{Z}[v]$ has a non-zero constant term. Let $\mu \in \Lambda_{n}$ be such that $z^{\prime} \in \mathfrak{R}(\mu)$ and write $z^{\prime}=\mathbf{w}_{\mu}(k, l)$ where $1 \leqslant k, l \leqslant d_{\mu}$. Note that, by the definition of $\mathcal{A}$, we have $\lambda \not \mu$. Now we obtain the following identity:

$$
v^{m+a(\mu)} \mathfrak{X}_{\mu}^{k l}\left(Z_{w}\right)=\sum_{z \in \mathfrak{S}_{n}} v^{m} f_{z}\left(v^{a(\mu)} \mathfrak{X}_{\mu}^{k l}\left(C_{z}\right)\right) .
$$

Let $z \in \mathfrak{S}_{n}$ be such that $z \in \mathfrak{R}(\nu)$ where $\lambda \triangleleft \nu$. By induction, $C_{z} \in \mathcal{N}^{\nu}$. Now Corollary [4.6 shows that, if $\mathfrak{X}_{\mu}\left(C_{z}\right) \neq 0$, then $\nu \unlhd \mu$ and so $\lambda \triangleleft \mu$, a contradiction. Hence, the above sum need only be extended over all elements $z \in \mathcal{A}$. But then we have $v^{m} f_{z} \in \mathbb{Z}[v]$ and so

$$
v^{m+a(\mu)} \mathfrak{X}_{\mu}^{k l}\left(Z_{w}\right) \equiv \sum_{z \in \mathcal{A}} v^{m} f_{z} \varepsilon_{z} c_{z, \mu}^{k l} \quad \bmod \mathfrak{p} .
$$

Now, we have $c_{z, \mu}^{k l}=0$ unless $z=z^{\prime}$; see Lemma 3.4. Hence we obtain

$$
v^{m+a(\mu)} \mathfrak{X}_{\mu}^{k l}\left(Z_{w}\right) \equiv v^{m} f_{z^{\prime}} \varepsilon_{z^{\prime}} c_{z^{\prime}, \mu}^{k l} \equiv \pm v^{m} f_{z^{\prime}} \quad \bmod \mathfrak{p}
$$

Since $v^{m} f_{z^{\prime}}$ has a non-zero constant term, we conclude that the above expression is not congruent to 0 modulo $\mathfrak{p}$. In particular, the left hand side is non-zero. Since $Z_{w} \in \mathcal{N}^{\lambda}$, we can now deduce that $\lambda \unlhd \mu$; see Corollary 4.6] Since we also have $\lambda \Varangle \mu$, we conclude that $\lambda=\mu$. Thus, we have reached the conclusion that

$$
v^{m}\left(v^{a(\lambda)} \mathfrak{X}_{\lambda}^{k l}\left(Z_{w}\right)\right) \not \equiv 0 \quad \bmod \mathfrak{p}
$$

Using Lemma 4.9] we now see that $m=0$. Thus, (1) is proved.

Claim (2). We have $f_{z}=0$ unless $\lambda \unlhd \lambda_{z}$.

To see this, let $\mu \in \Lambda_{n}$ be such that $\lambda \nexists \mu$. Assume, if possible, that there exists some $z^{\prime} \in \mathfrak{R}(\mu)$ such that $f_{z^{\prime}} \neq 0$. Let $1 \leqslant k, l \leqslant d_{\mu}$ be such that $z^{\prime}=\mathbf{w}_{\mu}(k, l) \in \mathfrak{R}(\mu)$. Now, as above, we see that

$$
v^{a(\mu)} \mathfrak{X}_{\mu}^{k l}\left(Z_{w}\right)=\sum_{z} f_{z}\left(v^{a(\mu)} \mathfrak{X}_{\mu}^{k l}\left(C_{z}\right)\right)
$$

where the sum need only be extended over all $z \in \mathfrak{S}_{n}$ such that $\lambda \unlhd \lambda_{z}$. By (1), we have $f_{z} \in \mathbb{Z}$ for all such elements $z$. Hence we obtain

$$
v^{a(\mu)} \mathfrak{X}_{\mu}^{k l}\left(Z_{w}\right) \in \mathcal{O} \quad \text { and } \quad v^{a(\mu)} \mathfrak{X}_{\mu}^{k l}\left(Z_{w}\right) \equiv \pm f_{z^{\prime}} \not \equiv 0 \quad \bmod \mathfrak{p},
$$

since $c_{z^{\prime}, \mu}^{k l}= \pm 1$ and $c_{z, \mu}^{k l}=0$ if $z \neq z^{\prime}$. In particular, we can now conclude that $\mathfrak{X}_{\mu}\left(Z_{w}\right) \neq 0$. But then Lemma 4.6 shows that $\lambda \unlhd \mu$, a contradiction. Thus, (2) is proved.

Claim (3). We have $\pm C_{w} \in Z_{w}+\hat{\mathcal{N}}^{\lambda}$. 
This is seen as follows. By (1) and (2), we can write

$$
Z_{w} \equiv \sum_{z \in \mathfrak{R}(\lambda)} f_{z} C_{z} \quad \bmod \quad\left\langle C_{y} \mid y \in \mathfrak{S}_{n}, \lambda \triangleleft \lambda_{y}\right\rangle_{A},
$$

where $f_{z} \in \mathbb{Z}$ for all $z \in \mathfrak{R}(\lambda)$. By induction, we have $C_{y} \in \hat{\mathcal{N}}^{\lambda}$ for any $y \in \mathfrak{S}_{n}$ such that $\lambda \triangleleft \lambda_{y}$. Thus, we have

$$
Z_{w} \equiv \sum_{z \in \mathfrak{R}(\lambda)} f_{z} C_{z} \quad \bmod \hat{\mathcal{N}}^{\lambda} \quad \text { where } f_{z} \in \mathbb{Z} .
$$

Now fix $z_{0} \in \mathfrak{R}(\lambda)$ and write $z_{0}=\mathbf{w}_{\lambda}(k, l)$ where $1 \leqslant k, l \leqslant d_{\lambda}$. In order to determine $f_{z_{0}}$, we multiply the above relation by $v^{a(\lambda)}$ and apply $\mathfrak{X}_{\lambda}^{k l}$. By Corollary 4.6] we have $\mathfrak{X}_{\lambda}(h)=0$ for all $h \in \hat{\mathcal{N}}^{\lambda}$. Hence we obtain

$$
v^{a(\lambda)} \mathfrak{X}_{\lambda}^{k l}\left(Z_{w}\right)=\sum_{z \in \mathfrak{R}(\lambda)} f_{z}\left(v^{a(\lambda)} \mathfrak{X}_{\lambda}^{k l}\left(C_{z}\right)\right) .
$$

Now let us take constant terms. On the left, we obtain \pm 1 if $k=i$ and $l=j$, and 0 otherwise; see Lemma 4.9. On the right, we obtain

$$
\sum_{z \in \mathfrak{R}(\lambda)} f_{z} \varepsilon_{z} c_{z, \lambda}^{k l}= \pm f_{z_{0}}
$$

Hence, we obtain $f_{z_{0}}= \pm 1$ if $z_{0}=w$ and $f_{z_{0}}=0$, otherwise; thus, (3) is proved and the proof of (a) is complete.

(b) Let $\mathcal{M}^{\lambda}$ be the $A$-submodule of $\mathcal{H}$ defined by the right hand side of the desired identity in (b). Let $\mu \in \Lambda_{n}$ be such that $\lambda \unlhd \mu$ and $w \in \mathfrak{R}(\mu)$. Since $Z_{w} \in \mathcal{N}^{\mu}$, we can deduce from (a) that $C_{w} \in \mathcal{N}^{\mu} \subseteq \mathcal{N}^{\lambda}$. Hence, we have

$$
\mathcal{M}^{\lambda} \subseteq \mathcal{N}^{\lambda}
$$

Now note that $|\mathbb{T}(\lambda)|=d_{\lambda}$; see Corollary 4.5. Hence, both $\mathcal{N}^{\lambda}$ and $\mathcal{M}^{\lambda}$ are free $A$-modules of the same rank, namely,

$$
\sum_{\substack{\mu \in \Lambda_{n} \\ \lambda \unlhd \mu}}|\mathfrak{R}(\lambda)|=\sum_{\substack{\mu \in \Lambda_{n} \\ \lambda \unlhd \mu}}\left|d_{\lambda}\right|^{2}=\sum_{\substack{\mu \in \Lambda_{n} \\ \lambda \unlhd \mu}}|\mathbb{T}(\lambda)|^{2} .
$$

Consequently, we have $K_{0} \otimes_{A} \mathcal{N}^{\lambda}=K_{0} \otimes_{A} \mathcal{M}^{\lambda}$, where $K_{0}$ is the field of fractions of $A$. So there exists some $0 \neq f \in A$ such that

$$
f \mathcal{N}^{\lambda} \subseteq \mathcal{M}^{\lambda} \subseteq \mathcal{N}^{\lambda}
$$

that is, $\mathcal{N}^{\lambda} / \mathcal{M}^{\lambda}$ is contained in the torsion part of $\mathcal{H} / \mathcal{M}^{\lambda}$. But, since the generators of $\mathcal{M}^{\lambda}$ can be extended to an $A$-basis of $\mathcal{H}$, the quotient $\mathcal{H} / \mathcal{M}^{\lambda}$ is a free $A$-module. Hence, we also have $\mathcal{N}^{\lambda} \subseteq \mathcal{M}^{\lambda}$, as desired.

Corollary 4.11. Let $\lambda \in \Lambda_{n}$ and $\mathfrak{s}, \mathfrak{t} \in \mathbb{T}(\lambda)$. Then there exists a unique element $w \in \mathfrak{R}(\lambda)$ such that

$$
\begin{aligned}
\tilde{y}_{\mathfrak{s t}}=\eta_{w} C_{w} & +v \mathbb{Z}[v] \text {-combination of elements } C_{y} \text { where } \lambda=\lambda_{y} \\
& +A \text {-combination of elements } C_{y} \text { where } \lambda \triangleleft \lambda_{y} .
\end{aligned}
$$


We have $w=\mathbf{w}_{\lambda}(i, j)$, where $1 \leqslant i, j \leqslant d_{\lambda}$ are such that $d(\mathfrak{s})=x_{i}$ and $d(\mathfrak{t})=x_{j}$.

Applying the ring involution $j: \mathcal{H} \rightarrow \mathcal{H}$ and using Corollary 4.3, we also obtain a relation with the original Murphy basis:

$$
\begin{aligned}
v^{-l\left(w_{\lambda}\right)} x_{\mathfrak{s t}}=\eta_{w}^{\prime} C_{w}^{\prime} & +v^{-1} \mathbb{Z}\left[v^{-1}\right] \text {-combination of elements } C_{y}^{\prime} \text { where } \lambda=\lambda_{y} \\
& +A \text {-combination of elements } C_{y}^{\prime} \text { where } \lambda \triangleleft \lambda_{y},
\end{aligned}
$$

where $\eta_{w}^{\prime}=\varepsilon_{d(\mathfrak{s})} \varepsilon_{d(\mathfrak{t})} \varepsilon_{w_{\lambda}} \varepsilon_{w} \eta_{w}= \pm 1$.

Proof. We begin with the following preliminary remarks. Let $x \in X_{\lambda}$. In the proof of Lemma 4.8 we have used [6, Prop. 3.3] to write $C_{x w_{\lambda}}$ as a linear combination of terms $T_{y} C_{w_{\lambda}}$ where $y \in X_{\lambda}$. But, the result in [loc. cit.] actually yields something stronger, namely, we have

$$
C_{x w_{\lambda}}=T_{x} C_{w_{\lambda}}+\sum_{\substack{y \in X_{\lambda} \\ y<x}} a_{x y} T_{y} C_{w_{\lambda}} \quad \text { where } \quad a_{x y} \in v \mathbb{Z}[v] .
$$

(Note that, in [6], we work with the $C^{\prime}$-basis; applying the ring involution $j: \mathcal{H} \rightarrow \mathcal{H}$ yields the above reformulation.) Inverting the above relations, we also obtain that

$$
T_{x} C_{w_{\lambda}}=C_{x w_{\lambda}}+\sum_{\substack{y \in X_{\lambda} \\ y<x}} b_{x y} C_{y w_{\lambda}} \quad \text { where } \quad b_{x y} \in v \mathbb{Z}[v] .
$$

Now assume that $x=x_{i}$ where $1 \leqslant i \leqslant d_{\lambda}$ is such that $d(\mathfrak{s})=x_{i}$. Let $y \in X_{\lambda}$ be such that $y<x$ and $b_{x y} \neq 0$. By Theorem 4.10(b), we have either $y w_{\lambda} \in \mathfrak{R}(\lambda)$ or $C_{y w_{\lambda}} \in \hat{\mathcal{N}}^{\lambda}$. Hence, using Lemma 3.10, we can write

$$
T_{x_{i}} C_{w_{\lambda}} \equiv C_{x_{i} w_{\lambda}}+\sum_{k=1}^{d_{\lambda}} b_{i k} C_{x_{k} w_{\lambda}} \quad \bmod \hat{\mathcal{N}}^{\lambda}
$$

where $b_{i k} \in v \mathbb{Z}[v]$. Applying the anti-involution $b: T_{x} \mapsto T_{x^{-1}}$, we can also write

$$
C_{w_{\lambda}} T_{x_{j}^{-1}} \equiv C_{w_{\lambda} x_{j}^{-1}}+\sum_{l=1}^{d_{\lambda}} b_{j l} C_{w_{\lambda} x_{l}^{-1}} \bmod \hat{\mathcal{N}}^{\lambda}
$$

where $b_{j l} \in v \mathbb{Z}[v]$ and $1 \leqslant j \leqslant d_{\lambda}$ is such that $d(\mathfrak{t})=x_{j}$. Consequently, we obtain that

$$
T_{x_{i}} C_{w_{\lambda}}^{2} T_{x_{j}^{-1}} \equiv C_{x_{i} w_{\lambda}} C_{w_{\lambda} x_{j}^{-1}}+\sum_{k, l=1}^{d_{\lambda}} c_{k l} C_{x_{k} w_{\lambda}} C_{w_{\lambda} x_{l}^{-1}} \quad \bmod \hat{\mathcal{N}}^{\lambda}
$$

where $c_{k l} \in v \mathbb{Z}[v]$. Using the identity in Lemma 2.8 and the definition of $Z_{w}$, we obtain that

$$
\tilde{y}_{\mathfrak{s t}} \equiv Z_{\mathbf{w}_{\lambda}(i, j)}+\sum_{k, l=1}^{d_{\lambda}} c_{k l} Z_{\mathbf{w}_{\lambda}(k, l)} \quad \bmod \hat{\mathcal{N}}^{\lambda}
$$


Finally, Theorem 4.10(a) yields

$$
\tilde{y}_{\mathfrak{s t}} \equiv \eta_{i j} C_{\mathbf{w}_{\lambda}(i, j)}+\sum_{k, l=1}^{d_{\lambda}} c_{k l} \eta_{k l} C_{\mathbf{w}_{\lambda}(k, l)} \bmod \hat{\mathcal{N}}^{\lambda}
$$

where we set $\eta_{k l}:=\eta_{y}$ if $y=\mathbf{w}_{\lambda}(k, l)$. Thus, the desired assertion holds where $w=\mathbf{w}_{\lambda}(i, j)$.

Example 4.12. Let $n=4$ and $\lambda=(3,1)$. We have $w_{(3,1)}=s_{1} s_{2} s_{1}$; the standard $(3,1)$-tableaux are given by

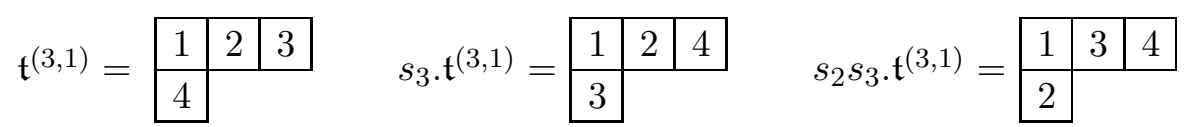

Thus, we have $\left\{d \in X_{(3,1)} \mid d \cdot \mathfrak{t}^{(3,1)} \in \mathbb{T}(1,3)\right\}=\left\{1, s_{3}, s_{2} s_{3}\right\}$. Given $\mathfrak{s}, \mathfrak{t} \in$ $\mathbb{T}(3,1)$, we write $\tilde{y}_{d(\mathfrak{s}), d(\mathfrak{t})}$ instead of $\tilde{y}_{\mathfrak{s t}}$. With this convention, the nine Murphy basis elements corresponding to pairs of standard $(3,1)$-tableaux are given as follows:

$$
\begin{aligned}
& \tilde{y}_{1,1}=\underline{C}_{s_{1} s_{2} s_{1}}, \\
& \tilde{y}_{1, s_{3}}=v C_{s_{1} s_{2} s_{1}}+\underline{C}_{s_{1} s_{2} s_{1} s_{3}}, \\
& \tilde{y}_{1, s_{2} s_{3}}=v C_{s_{1} s_{2} s_{1} s_{3}}+\underline{C}_{s_{1} s_{2} s_{1} s_{3} s_{2}} \\
& \tilde{y}_{s_{3}, 1}=v C_{s_{1} s_{2} s_{1}}+\underline{C}_{s_{1} s_{3} s_{2} s_{1}}, \\
& \tilde{y}_{s_{3}, s_{3}}=v^{2} C_{s_{1} s_{2} s_{1}}+v C_{s_{1} s_{2} s_{1} s_{3}}+v C_{s_{1} s_{3} s_{2} s_{1}}+\underline{C}_{s_{1} s_{2} s_{3} s_{2} s_{1}}, \\
& \tilde{y}_{s_{3}, s_{2} s_{3}}=v^{2} C_{s_{1} s_{2} s_{1} s_{3}}+\underline{C}_{s_{1} s_{2} s_{3} s_{2}}+v C_{s_{1} s_{2} s_{1} s_{3} s_{2}} \\
& \quad+v C_{s_{1} s_{2} s_{3} s_{2} s_{1}}+C_{s_{1} s_{2} s_{1} s_{3} s_{2} s_{1}}, \\
& \tilde{y}_{s_{2} s_{3}, 1}=v C_{s_{1} s_{3} s_{2} s_{1}}+\underline{C}_{s_{2} s_{1} s_{3} s_{2} s_{1}}, \\
& \tilde{y}_{s_{2} s_{3}, s_{3}}=v^{2} C_{s_{1} s_{3} s_{2} s_{1}}+\underline{C}_{s_{2} s_{3} s_{2} s_{1}}+v C_{s_{1} s_{2} s_{3} s_{2} s_{1}} \\
& \quad+v C_{s_{2} s_{1} s_{3} s_{2} s_{1}}+C_{s_{1} s_{2} s_{1} s_{3} s_{2} s_{1}}, \\
& \tilde{y}_{s_{2} s_{3}, s_{2} s_{3}}= \underline{C}_{s_{2} s_{3} s_{2}}+v C_{s_{1} s_{2} s_{3} s_{2}}+v C_{s_{2} s_{3} s_{2} s_{1}} \\
& \quad+v^{2} C_{s_{1} s_{2} s_{3} s_{2} s_{1}}+\left(v-v^{-1}\right) C_{s_{1} s_{2} s_{1} s_{3} s_{2} s_{1}}
\end{aligned}
$$

In each case, we have underlined the Kazhdan-Lusztig basis element which corresponds to the Murphy basis element as in Corollary 4.11. These examples show, in particular, that the coefficient of $C_{y}$, where $\lambda \triangleleft \lambda_{y}$, may involve negative powers of $v$.

\section{Applications to the Kazhdan-Lusztig Cells in $\mathfrak{S}_{n}$}

We keep the setting of the previous sections. In particular, recall the partition

$$
\mathfrak{S}_{n}=\coprod_{\lambda \in \Lambda_{n}} \mathfrak{R}(\lambda) \quad \text { where } \quad w_{\lambda} \in \mathfrak{R}(\lambda)
$$


see Example 3.8. By Remark 3.5. we already know that each set $\mathfrak{R}(\lambda)$ is contained in a two-sided cell of $\mathfrak{S}_{n}$. Now we can prove the following result.

Theorem 5.1. Let $\lambda, \mu \in \Lambda_{n}$ and $x \in \mathfrak{R}(\lambda), y \in \mathfrak{R}(\mu)$. Then we have

$$
x \leqslant \mathcal{L R} y \quad \Leftrightarrow \quad \mu \unlhd \lambda .
$$

In particular, $w_{\lambda} \leqslant \mathcal{L R} w_{\mu}$ if and only if $\mu \unlhd \lambda$. The sets $\mathfrak{R}(\lambda), \lambda \in \Lambda_{n}$, are precisely the two-sided cells of $\mathfrak{S}_{n}$.

Proof. If $\mu \unlhd \lambda$, then $w_{\lambda} \leqslant \mathcal{L R} w_{\mu}$ by Remark 3.11. Since each of the sets $\mathfrak{R}(\lambda)$ and $\mathfrak{R}(\mu)$ is contained in a two-sided cell, we conclude that $x \leqslant \mathcal{L R} y$. Conversely, assume that $x \leqslant \mathcal{L R} y$. We may assume without loss of generality that $x \leftarrow_{\mathcal{L}} y$ or $x \leftarrow_{\mathcal{R}} y$. Let us first assume that $x \leftarrow_{\mathcal{L}} y$. This means that exists some $h \in \mathcal{H}$ such that $C_{x}$ occurs in $h C_{y}$ (expressed in the $C$-basis). Since $y \in \mathcal{R}(\mu)$, we have $C_{y} \in \mathcal{N}^{\mu}$ by Theorem 4.10(a). Hence we also have $h C_{y} \in \mathcal{N}^{\mu}$ since $\mathcal{N}^{\mu}$ is a two-sided ideal. But then Theorem 4.10(b) shows that $h C_{y}$ can be written as an $A$-linear combination of elements $C_{z}$ where $\mu \unlhd \lambda_{z}$. Since $C_{x}$ occurs in that linear combination, we conclude that $\mu \unlhd \lambda$, as desired. The argument in the case where $x \leftarrow_{\mathcal{R}} y$ is completely analogous. The statement concerning the two-sided cells is now clear.

The equivalence " $w_{\lambda} \leqslant \mathcal{L R}_{\mathcal{R}} w_{\mu} \Leftrightarrow \mu \unlhd \lambda$ " is not a new result. However, as far as we are aware of, all the previously known proofs for the implication " $\Rightarrow$ " rely on the geometric interpretation of the Kazhdan-Lusztig basis and the resulting "positivity properties". See, for example, Du-Parshall-Scott [4. 2.13.1], where this is deduced from a result of Lusztig-Xi [15, 3.2].

Lemma 5.2. Let $\lambda \in \mathfrak{R}(\lambda)$ and $1 \leqslant j \leqslant d_{\lambda}$. Then

$$
V_{j}^{\lambda}:=\left\langle C_{\mathbf{w}_{\lambda}(i, j)}+\hat{\mathcal{N}}^{\lambda} \mid 1 \leqslant i \leqslant d_{\lambda}\right\rangle_{A} \subseteq \mathcal{N}^{\lambda} / \hat{\mathcal{N}}^{\lambda}
$$

is a left $\mathcal{H}$-module, free over $A$ of rank $d_{\lambda}$. We have

$$
\mathcal{N}^{\lambda} / \hat{\mathcal{N}}^{\lambda}=V_{1}^{\lambda} \oplus \cdots \oplus V_{d_{\lambda}}^{\lambda} .
$$

Proof. First note that, by Theorem 4.10, $\mathcal{N}^{\lambda} / \hat{\mathcal{N}}^{\lambda}$ is free over $A$, with a basis given by the residue classes of the elements $C_{w}$ where $w \in \mathfrak{R}(\lambda)$. This already shows that each $V_{j}^{\lambda}$ is free over $A$ of rank $d_{\lambda}$, and that $\mathcal{N}^{\lambda} / \hat{\mathcal{N}}^{\lambda}$ is the direct sum of all $V_{j}^{\lambda}$. It remains to prove that $V_{j}^{\lambda}$ is a left $\mathcal{H}$-module. For this purpose, we must prove that, for any $h \in \mathcal{H}$ and $1 \leqslant i, k \leqslant d_{\lambda}$, there exist $r_{h}^{j}(i, k) \in A$ such that

$$
h C_{\mathbf{w}_{\lambda}(i, j)} \equiv \sum_{k=1}^{d_{\lambda}} r_{h}^{j}(i, k) C_{\mathbf{w}_{\lambda}(k, j)} \bmod \hat{\mathcal{N}}^{\lambda} .
$$

First we prove this in the case where $j=1$. Then

$$
\mathbf{w}_{\lambda}(i, 1)=x_{i} w_{\lambda} \quad \text { where } x_{i} \in X_{\lambda}
$$

see Remark 3.9. Now, by Lemma 2.8(c), the $A$-module

$$
\left\langle C_{x w_{\lambda}} \mid x \in X_{\lambda}\right\rangle_{A}
$$


is a left ideal in $\mathcal{H}$. Hence, for any $h \in \mathcal{H}$, we can write

$$
h C_{x_{i} w_{\lambda}}=\sum_{x \in X_{\lambda}} r_{h}(i, x) C_{x w_{\lambda}} \quad \text { where } r_{h}(i, x) \in A .
$$

Now let $x \in X_{\lambda}$ and $\mu \in \Lambda_{n}$ be such that $x w_{\lambda} \in \mathfrak{R}(\mu)$. Then, by Lemma 3.10. we have either $\lambda \triangleleft \mu$ or $x=x_{k}$ for some $k \in\left\{1, \ldots, d_{\lambda}\right\}$. By Theorem 4.10, the former condition implies that $C_{x w_{\lambda}} \in \hat{\mathcal{N}}^{\lambda}$. Consequently, we have

$$
h C_{x_{i} w_{\lambda}} \equiv \sum_{k=1}^{d_{\lambda}} r_{h}\left(i, x_{k}\right) C_{x_{k} w_{\lambda}} \quad \bmod \hat{\mathcal{N}}^{\lambda}
$$

Thus, the desired assertion holds where we set $r_{h}^{1}(i, k):=r_{h}\left(i, x_{k}\right)$.

Now let $j>1$. By Theorem 4.10, there exist signs $\eta_{i j}= \pm 1$ such that

$$
C_{x_{i} w_{\lambda}} C_{w_{\lambda} x_{j}^{-1}} \equiv \eta_{i j} C_{\mathbf{w}_{\lambda}(i, j)} \quad \bmod \hat{\mathcal{N}}^{\lambda} \quad \text { for } 1 \leqslant i \leqslant d_{\lambda} .
$$

We shall now set

$$
r_{h}^{j}(i, k):=\eta_{i j} \eta_{k j} r_{h}^{1}(i, k) \quad \text { for } 1 \leqslant i, k \leqslant d_{\lambda} .
$$

Then, since $\hat{\mathcal{N}}^{\lambda}$ is a two-sided ideal, we obtain

$$
\begin{aligned}
h C_{\mathbf{w}_{\lambda}(i, j)} & \equiv \eta_{i j}\left(h C_{x_{i} w_{\lambda}}\right) C_{w_{\lambda} x_{j}^{-1}} \\
& \equiv \eta_{i j} \sum_{k=1}^{d_{\lambda}} r_{h}^{1}(i, k) C_{x_{k} w_{\lambda}} C_{w_{\lambda} x_{j}^{-1}} \\
& \equiv \eta_{i j} \sum_{k=1}^{d_{\lambda}} \eta_{k i} r_{h}^{1}(i, k) C_{\mathbf{w}_{\lambda}(k, j)} \\
& \equiv \sum_{k=1}^{d_{\lambda}} r_{h}^{j}(i, k) C_{\mathbf{w}_{\lambda}(k, j)}
\end{aligned}
$$

as desired, where all of the above congruences are taken modulo $\hat{\mathcal{N}}^{\lambda}$.

Theorem 5.3. Let $x, y \in \mathfrak{S}_{n}$. Then we have the following implication:

$$
x \leqslant_{\mathcal{L}} y \quad \text { and } \quad x \sim_{\mathcal{L R}} y \quad \Rightarrow \quad x \sim_{\mathcal{L}} y .
$$

Proof. Let $x, y \in \mathfrak{S}_{n}$ be such that $x \leqslant_{\mathcal{L}} y$ and $x \sim_{\mathcal{L} \mathcal{R}} y$. Since the relation $\leqslant_{\mathcal{L}}$ is defined as the transitive closure of $\leftarrow_{\mathcal{L}}$, it will be sufficient to consider the special case where $x \leftarrow \mathcal{L} y$. Now, since $x \sim_{\mathcal{L} R} y$, we have $x, y \in \mathfrak{R}(\lambda)$ for some $\lambda \in \mathfrak{R}(\lambda)$; see Theorem [5.1] Thus, we have $y=\mathbf{w}_{\lambda}(i, j)$ where $1 \leqslant i, j \leqslant d_{\lambda}$. By Lemma 5.2 , we have

$$
h C_{y} \equiv h C_{\mathbf{w}_{\lambda}(i, j)} \equiv \sum_{k=1}^{d_{\lambda}} r_{h}^{j}(i, k) C_{\mathbf{w}_{\lambda}(k, j)} \bmod \hat{\mathcal{N}}^{\lambda},
$$

where $r_{h}^{j}(i, k) \in A$. By Theorem 4.10 and Theorem [5.1, $\hat{\mathcal{N}}^{\lambda}$ is spanned by elements $C_{w}$ where $w \leqslant \mathcal{L R} y$ but $w \chi_{\mathcal{L R}} y$. Hence, our hypothesis that 
$C_{x}$ occurs in the decomposition of $h C_{y}$ implies that either $x=\mathbf{w}_{\lambda}(k, j)$ for some $1 \leqslant k \leqslant d_{\lambda}$ or $x \nsim_{\mathcal{L R}} y$. Hence, we must have $x=\mathbf{w}_{\lambda}(k, j)$ and so $x \sim_{\mathcal{L}} y$ by Remark 3.5 .

Again, the above result is not new (and the conclusion is known to hold for more general types of Coxeter groups). But, even for the symmetric group $\mathfrak{S}_{n}$, it was first proved by Lusztig [12, Lemma 4.1] using the (deep) connection between cells and primitive ideals in universal envelopping algebras via the main conjecture of Kazhdan-Lusztig 10. Another proof, applicable to finite and affine Weyl groups, was given by Lusztig 14, using the geometric interpretation of the Kazhdan-Lusztig basis and the resulting "positivity properties".

The following result was first obtained by Kazhdan-Lusztig 10, Theorem 1.4], as a consequence of the combinatorial description of the left cells in terms of the Robinson-Schensted correspondence. (We will come back to the latter point in Theorem [5.6.)

Theorem 5.4. For each left cell $\mathfrak{C}$ of $\mathfrak{S}_{n}$, we have $\chi_{\mathfrak{C}} \in \operatorname{Irr}\left(\mathcal{H}_{K}\right)$. Furthermore, two left cells $\mathfrak{C}, \mathfrak{C}^{\prime}$ afford the same character if and only if $\mathfrak{C}, \mathfrak{C}^{\prime \prime} \subseteq \mathfrak{R}(\lambda)$ for some $\lambda \in \Lambda_{n}$. The total number of left cells equals $\sum_{\lambda \in \Lambda_{n}} d_{\lambda}$.

Proof. For $\lambda \in \Lambda_{n}$ and $1 \leqslant j \leqslant d_{\lambda}$, we set

$$
{ }^{j} \mathcal{C}_{\lambda}:=\left\{\mathbf{w}_{\lambda}(i, j) \mid 1 \leqslant i \leqslant d_{\lambda}\right\} \subseteq \mathfrak{R}(\lambda) .
$$

By Remark 3.5 the above set is contained in a left cell of $\mathfrak{S}_{n}$. We claim that ${ }^{j} \mathcal{C}_{\lambda}$ is a left cell. Since $\leqslant_{\mathcal{L}}$ is defined as the transitive closure of the relation $\leftarrow \mathcal{L}$, it is enough to prove the following statement.

Let $y \in{ }^{j} \mathcal{C}_{\lambda}$ and $h \in \mathcal{H}$. Then $h C_{y}$ is a linear combination of basis elements $C_{x}$ where $x \in \mathfrak{C}_{j}^{\lambda}$ or $x<_{\mathcal{L R}} y$.

This is proved as follows. By Lemma 5.2 we can write $h C_{y}$ as a linear combination of basis elements $C_{x}$ where $x \in{ }^{j} \mathcal{C}_{\lambda}$, and some element of $\hat{\mathcal{N}}^{\lambda}$. But, by Theorem 4.10 and Theorem [5.1] $\hat{\mathcal{N}}^{\lambda}$ is spanned by basis elements $C_{w}$ where $w<_{\mathcal{L R}} y$. This yields the above statement. Thus, ${ }^{j} \mathcal{C}_{\lambda}$ is a left cell, as claimed. Now it is clear that we have a partition

$$
\mathfrak{S}_{n}=\coprod_{\lambda \in \Lambda_{n}} \coprod_{j=1}^{d_{\lambda}}{ }^{j} \mathcal{C}_{\lambda} .
$$

Hence, the sets ${ }^{j} \mathcal{C}_{\lambda}$ are precisely the left cells of $\mathfrak{S}_{n}$. The remaining statements concerning the characters of the left cell representations now follow by a standard counting argument. Indeed, since $\mathcal{H}_{K}$ is semisimple, the above partition yields an isomorphism of left $\mathcal{H}_{K}$-modules

$$
\mathcal{H}_{K} \cong \bigoplus_{\lambda \in \Lambda_{n}} \bigoplus_{j=1}^{d_{\lambda}}\left[{ }^{j} \mathcal{C}_{\lambda}\right]_{K} .
$$


Thus, $\mathcal{H}_{K}$ (regarded as a left $\mathcal{H}_{K}$-module) has a decomposition into a direct sum with $\sum_{\lambda} d_{\lambda}$ terms. Now recall that the latter number is the sum of the dimensions of all irreducible representations of $\mathcal{H}_{K}$ (up to equivalence). Hence, by Wedderburn's Theorem, $\sum_{\lambda} d_{\lambda}$ is the maximum number of terms in a direct sum decomposition of $\mathcal{H}_{K}$. Consequently, each direct summand $\left[{ }^{j} \mathcal{C}_{\lambda}\right]_{K}$ must be a simple $\mathcal{H}_{K}$-module. Thus, we have shown that $[\mathfrak{C}]_{K}$ is a simple $\mathcal{H}_{K}$-module for any left cell $\mathfrak{C}$. Finally, we claim that

$$
\chi_{\lambda}=\text { character afforded by }\left[{ }^{j} \mathcal{C}_{\lambda}\right]_{K} \text {. }
$$

Indeed, since ${ }^{j} \mathcal{C}_{\lambda} \subseteq \mathfrak{R}(\lambda)$, Proposition 3.3 shows that $\chi_{\lambda}$ occurs in the character afforded by $\left[{ }^{j} \mathcal{C}_{\lambda}\right]_{K}$. Since the latter is irreducible, we have equality, as desired. We conclude that two left cells afford the same character if and only if these two left cells are both contained in $\mathfrak{R}(\lambda)$ for some $\lambda$.

Let us recall some basic facts concerning the Robinson-Schensted correspondence. We use Knuth [11, §5.1.4] as a reference. Recall that, for any $\lambda \in \Lambda_{n}$, we denote by $\mathbb{T}(\lambda)$ the set of all standard $\lambda$-tableaux. Then the Robinson-Schensted correspondence defines a bijection

$$
\coprod_{\lambda \in \Lambda_{n}}(\mathbb{T}(\lambda) \times \mathbb{T}(\lambda)) \rightarrow \mathfrak{S}_{n}
$$

If $\mathfrak{s}, \mathfrak{t} \in \mathbb{T}(\lambda)$, we denote by $\pi_{\lambda}(\mathfrak{s}, \mathfrak{t})$ the corresponding element of $\mathfrak{S}_{n}$. Given $w \in \mathfrak{S}_{n}$, the pair $(\mathfrak{s}, \mathfrak{t})$ such that $w=\pi_{\lambda}(\mathfrak{s}, \mathfrak{t})$ can be constructed explicitly by the "insertion algorithm" [11, p. 49]. The tableau $\mathfrak{s}$ is obtained by "inserting" the numbers from the sequence $(w \cdot 1, w .2, \ldots, w \cdot n)$ into an initially empty tableau; the tableau $\mathfrak{t}$ "keeps the record" of the order in which the positions in $\mathfrak{s}$ have been filled. For example, applying the insertion algorithm to the element $w_{\lambda}$ where $\lambda \in \Lambda_{n}$, we obtain

$$
w_{\lambda}=\pi_{\lambda^{*}}\left(\mathfrak{t}^{\lambda^{*}}, \mathfrak{t}^{\lambda^{*}}\right)
$$

where $\lambda^{*}$ denotes the conjugate partition.

We shall need the following property of the Robinson-Schensted correspondence.

Proposition 5.5 (Knuth). Let $\lambda \in \Lambda_{n}$ and $\mathfrak{s}, \mathfrak{s}^{\prime}, \mathfrak{t}, \mathfrak{t}^{\prime} \in \mathbb{T}(\lambda)$. We set

$$
w:=\pi_{\lambda}(\mathfrak{s}, \mathfrak{t}) \quad \text { and } \quad w^{\prime}:=\pi_{\lambda}\left(\mathfrak{s}^{\prime}, \mathfrak{t}^{\prime}\right) .
$$

Then we have $\mathfrak{s}=\mathfrak{s}^{\prime}$ if and only if $w, w^{\prime}$ are linked by a finite sequence of "star operations", that is, there exists a sequence $w=y_{0}, y_{1}, \ldots, y_{k}=w^{\prime}$ of elements $y_{i} \in \mathfrak{S}_{n}$ such that $y_{i-1} \approx y_{i}$ for all $i$, where $\approx$ is defined in (2.6).

For the proof, see Exc. 4 in [11, §5.1.4]. Note that Knuth shows that we have $\mathfrak{s}=\mathfrak{s}^{\prime}$ if and only if the permutations $w, w^{\prime}$ can be transformed to each other by a finite sequence of so-called "admissible transformations". It is readily checked that the latter condition is equivalent to the fact that $w, w^{\prime}$ are linked by a finite sequence of "star operations", using the characterisation of $l(w)$ as the number of pairs $(i, j)$ such that $1 \leqslant i<j \leqslant n$ and $w(j)>w(i)$. See also [1, §3.2] for more details. 
Now we can prove the following result. The statements in (a) and (b) are due to Kazhdan-Lusztig [10, §5], but the proof given there is quite sketchy. A complete, self-contained proof, based on the methods in [10, §4], is given by Ariki [1. The proof that we give here is different as far as the (more difficult) implications " $\Rightarrow$ " are concerned. Note also that (c) is neither proved in [10] nor in [1].

Corollary 5.6. Let $w, w^{\prime} \in \mathfrak{S}_{n}$. Let $\lambda, \mu \in \Lambda_{n}$ be such that $w=\pi_{\lambda}(\mathfrak{s}, \mathfrak{t})$ where $\mathfrak{s}, \mathfrak{t} \in \mathbb{T}(\lambda)$ and $w^{\prime}=\pi_{\mu}\left(\mathfrak{s}^{\prime}, \mathfrak{t}^{\prime}\right)$ where $\mathfrak{s}^{\prime}, \mathfrak{t}^{\prime} \in \mathbb{T}(\mu)$. Then the following hold:

$$
\begin{aligned}
w \sim_{\mathcal{R}} w^{\prime} & \Leftrightarrow \quad \lambda=\mu \text { and } \mathfrak{s}=\mathfrak{s}^{\prime}, \\
w \sim_{\mathcal{L}} w^{\prime} & \Leftrightarrow \quad \lambda=\mu \text { and } \mathfrak{t}=\mathfrak{t}^{\prime}, \\
w \sim_{\mathcal{L R}} w^{\prime} & \Leftrightarrow \quad \lambda=\mu .
\end{aligned}
$$

In particular, the intersection of a left cell and a right cell is either empty or a singleton set. Furthermore, for any $\lambda \in \Lambda_{n}$, we have

$$
\mathfrak{R}(\lambda)=\left\{\pi_{\lambda^{*}}(\mathfrak{u}, \mathfrak{v}) \mid \mathfrak{u}, \mathfrak{v} \in \mathbb{T}\left(\lambda^{*}\right)\right\},
$$

where $\lambda^{*}$ denotes the conjugate partition.

Proof. First note that $d_{\lambda}=|\mathbb{T}(\lambda)|$ for any $\lambda \in \Lambda_{n}$; see Corollary 4.5.

(a) Let $\lambda \in \Lambda_{n}$ and $\mathfrak{s} \in \mathbb{T}(\lambda)$. By Proposition [5.5] and (2.6) (a), the set

$$
\mathbb{T}(\lambda, \mathfrak{s}):=\left\{\pi_{\lambda}(\mathfrak{s}, \mathfrak{t}) \mid \mathfrak{t} \in \mathbb{T}(\lambda)\right\}
$$

is contained in a right cell. Now note that the family of sets

$$
\left\{\mathbb{T}(\lambda, \mathfrak{s}) \mid \lambda \in \Lambda_{n}, \mathfrak{s} \in \mathbb{T}(\lambda)\right\}
$$

forms a partition of $\mathfrak{S}_{n}$, and that there are $\sum_{\lambda} d_{\lambda}$ pieces in that partition. On the other hand, by Theorem [5.4 the latter number also equals the number of all left cells of $\mathfrak{S}_{n}$, and that number is the same as the number of all right cells. Hence each set $\mathbb{T}(\lambda, \mathfrak{s})$ must be a right cell.

(b) We have $\pi_{\lambda}(\mathfrak{s}, \mathfrak{t})^{-1}=\pi_{\lambda}(\mathfrak{t}, \mathfrak{s})$ for all $\lambda \in \Lambda_{n}$ and $\mathfrak{s}, \mathfrak{t} \in \mathbb{T}(\lambda)$; see Theorem B in [11, §5.1.4]. Hence the assertion follows from (a).

(c) Let $\lambda \in \Lambda_{n}$. Then, by (a) and (b), the set $\left\{\pi_{\lambda}(\mathfrak{s}, \mathfrak{t}) \mid \mathfrak{s}, \mathfrak{t} \in \mathbb{T}(\lambda)\right\}$ is contained in a two-sided cell of $\mathfrak{S}_{n}$. Using Theorem 5.1. we can now argue as in the proof of (a) to conclude that the above set is a two-sided cell.

Finally, consider the statement concerning $\mathfrak{R}(\lambda)$. By Remark 3.9. we have $w_{\lambda} \in \mathfrak{R}(\lambda)$. On the other hand, applying the "insertion algorithm" to $w_{\lambda}$, we see that $w_{\lambda}=\pi_{\lambda^{*}}\left(\mathfrak{u}_{0}, \mathfrak{v}_{0}\right)$ for some standard $\lambda^{*}$-tableaux $\mathfrak{u}_{0}, \mathfrak{v}_{0}$. Now Theorem 5.1 and (c) imply that $\mathfrak{R}(\lambda)=\left\{\pi_{\lambda^{*}}(\mathfrak{u}, \mathfrak{v}) \mid \mathfrak{u}, \mathfrak{v} \in \mathbb{T}\left(\lambda^{*}\right)\right\}$.

Remark 5.7. Once the above result is established, we can also identify the elements $x_{i}$ in Remark [3.9] Indeed, for $\lambda \in \Lambda_{n}$, the set

$$
{ }^{1} \mathcal{C}_{\lambda}=\left\{x_{i} w_{\lambda} \mid 1 \leqslant i \leqslant d_{\lambda}\right\}
$$


is the left cell containing $w_{\lambda}$. Then the Robinson-Schensted correspondence shows that

$$
{ }^{1} \mathcal{C}_{\lambda}=\left\{d(\mathfrak{s}) w_{\lambda} \mid \mathfrak{s} \in \mathbb{T}(\lambda)\right\}
$$

see [19, Lemma 3.3] and the references there. We also remark here that, in 19. Theorem 3.5], McDonough and Pallikaros construct an explicit isomorphism of $\mathcal{H}$-modules from $\left[{ }^{1} \mathcal{C}_{\lambda}\right]_{A}$ onto the Specht module $\tilde{\mathcal{S}}^{\lambda^{*}}$, where $\lambda^{*}$ is the conjugate partition.

Corollary 5.8. Let $\lambda \in \Lambda_{n}$ and $\mathfrak{C}, \mathfrak{C}_{1}$ be two left cells contained in $\mathfrak{R}(\lambda)$. Then there is a unique bijection $\mathfrak{C} \stackrel{\sim}{\rightarrow} \mathfrak{C}_{1}, x \mapsto x_{1}$, such that the following conditions are satisfied:

$$
\begin{array}{cl}
x \sim_{\mathcal{R}} x_{1} & \text { for all } x \in \mathfrak{C}, \\
h_{w, x, y}=h_{w, x_{1}, y_{1}} & \text { for all } x, y \in \mathfrak{C} \text { and } w \in \mathfrak{S}_{n} .
\end{array}
$$

Thus, the $\mathcal{H}$-modules $[\mathfrak{C}]_{A}$ and $\left[\mathfrak{C}_{1}\right]_{A}$ are not only isomorphic, but they even afford exactly the same matrix representations with respect to the standard bases of $[\mathfrak{C}]_{A}$ and $[\mathfrak{C}]_{A}$, respectively.

Proof. This follows by the argument in [10, §5]. Indeed, by Corollary [5.6 and Proposition [5.5] $\mathfrak{C}$ and $\mathfrak{C}_{1}$ can be linked by a finite sequence of star operations. Hence the assertions follow from (2.6).

Finally, we turn to the properties (P1)-(P15) in (2.4). Recall that, for any $w \in \mathfrak{S}_{n}$, we defined $\alpha_{w}:=a(\lambda)=l\left(w_{\lambda}\right)$, where $\lambda \in \Lambda_{n}$ is such that $w \in \mathfrak{R}(\lambda)$; see Definition 4.7

Lemma 5.9. Let $x, y \in \mathfrak{S}_{n}$ and assume that $x \leqslant \mathcal{L R} y$. Then we have $\alpha_{y} \leqslant \alpha_{x}$, with equality only if $x \sim_{\mathcal{L R}} y$.

Proof. Let $\lambda, \mu \in \Lambda_{n}$ be such that $x \in \mathfrak{R}(\lambda), y \in \mathfrak{R}(\mu)$. By Corollary 5.1 we have $\mu \unlhd \lambda$. This implies $a(\mu) \leqslant a(\lambda)$ with equality only if $\lambda=\mu$; see, for example, [8, (5.4.2) and Exc. 5.6]. Thus, we have $\alpha_{y} \leqslant \alpha_{x}$, with equality only if $\lambda=\mu$. Finally, by Theorem [5.1] we have $x \sim_{\mathcal{L R}} y$ if $\lambda=\mu$.

Theorem 5.10. We have $\mathbf{a}(w)=\alpha_{w}$ for any $w \in \mathfrak{S}_{n}$. Furthermore, the properties (P1)-(P15) in (2.4) hold, where the set of "distinguished involutions" is given by

$$
\mathcal{D}=\left\{z \in \mathfrak{S}_{n} \mid z^{2}=1\right\} \text {. }
$$

The constants $\gamma_{x, y, z}$ are given as follows. Let $x, y, z \in \mathfrak{S}_{n}$ and $d \in \mathcal{D}$ be such that $y^{-1} \sim_{\mathcal{L}} d$. Then we have

$$
\gamma_{x, y, z}=\left\{\begin{array}{cl}
n_{d} & \text { if } x \sim_{\mathcal{L}} y^{-1}, y \sim_{\mathcal{L}} z^{-1}, z \sim_{\mathcal{L}} x^{-1}, \\
0 & \text { otherwise. }
\end{array}\right.
$$

Furthermore, $n_{d}=1$ for any $d \in \mathcal{D}$. Lusztig's ring $J$, see 2.4), is isomorphic to the direct sum of the matrix rings $M_{d_{\lambda}}(\mathbb{Z})\left(\lambda \in \Lambda_{n}\right)$. 
Proof. First we determine a $(w)$ and show that (P1)-(P15) hold. Since condition (*) in Lemma 3.4 is satisfied, we can apply the results in [7, §4]. By Lemma [5.9, the hypotheses of [7, Lemma 4.4] are satisfied. Hence we have $\mathbf{a}(w)=\alpha_{w}$ for any $w \in \mathfrak{S}_{n}$. Once the identity $\mathbf{a}(w)=\alpha_{w}$ is established, Lemma 5.9 shows that (P4) and (P11) hold.

But then we can also apply [7, Lemma 4.6] and this yields (P1), (P2), (P3), (P5), (P6), (P7), (P8) and (P14). Since all of the above properties hold for any parabolic subgroup of $\mathfrak{S}_{n},(\mathrm{P} 12)$ also holds; see the argument in [17. 14.12]. By Theorem 5.4, we have $\chi_{\mathfrak{C}} \in \operatorname{Irr}\left(\mathcal{H}_{K}\right)$ for any left cell $\mathfrak{C}$. Hence [7. Lemma 4.8] yields that (P13) holds and that $\mathcal{D}=\left\{z \in \mathfrak{S}_{n} \mid z^{2}=1\right\}$. By [7. Remark 4.10], we have

$$
\gamma_{x, y, z}=\left\{\begin{array}{cl} 
\pm 1 & \text { if } x \sim_{\mathcal{L}} y^{-1}, y \sim_{\mathcal{L}} z^{-1}, z \sim_{\mathcal{L}} x^{-1} \\
0 & \text { otherwise. }
\end{array}\right.
$$

The statement about the structure of $J$ is contained in [7, Lemma 4.9].

Next we show that (P9), (P10) hold. Now, by [17 14.10], property (P10) is a formal consequence of (P9). To prove (P9), let $x, y \in \mathfrak{S}_{n}$ be such that $x \leqslant_{\mathcal{L}} y$ and $\mathbf{a}(x)=\mathbf{a}(y)$. By $(\mathrm{P} 11)$, we obtain $x \sim_{\mathcal{L R}} y$. Hence Theorem 5.3 implies $x \sim_{\mathcal{L}} y$, as desired.

It remains to consider (P15). Since (P4), (P9), (P10) are already known to hold, (P15) can be reformulated as explained in [17, 14.15]. But then we can argue as in [17, 15.7] to conclude that (P15) hold. (Note that we are in the case of equal parameters; the "positivity properties" are not required in 17. 15.7].) Thus, we have proved (P1)-(P15) for $W=\mathfrak{S}_{n}$.

Next, let $x, y, z \in \mathfrak{S}_{n}$ and $d \in \mathcal{D}$ be such that $x \sim_{\mathcal{L}} y^{-1}, y \sim_{\mathcal{L}} z^{-1}$, $z \sim_{\mathcal{L}} x^{-1}$ and $y^{-1} \sim_{\mathcal{L}} d$. We show that $\gamma_{x, y, z}=n_{d}$. Now, by Theorem 5.1 we have $x, y, z \in \mathfrak{R}(\lambda)$ for some $\lambda \in \mathfrak{R}(\lambda)$. Furthermore, there exist $1 \leqslant$ $i, j \leqslant d_{\lambda}$ such that

$$
x=\mathbf{w}_{\lambda}(i, j), \quad y=\mathbf{w}_{\lambda}(j, k), \quad z=\mathbf{w}_{\lambda}(k, i), \quad d=\mathbf{w}_{\lambda}(j, j) .
$$

(See Remark 3.5 and note that, by the proof of Theorem [5.4 the sets ${ }^{j} \mathcal{C}_{\lambda}$ defined there are precisely the left cells contained in $\mathfrak{R}(\lambda)$.) By Remark 3.5. we also have $x \sim \mathcal{L} d$. So (P5), (P7), (P13) show that

$$
\gamma_{x, d, x^{-1}}=\gamma_{x^{-1}, x, d}=n_{d}= \pm 1 \text {. }
$$

Now, since $d=d^{-1} \sim_{\mathcal{R}} y, x \sim_{\mathcal{R}} z^{-1}$ and $y \sim_{\mathcal{L}} z^{-1}$, we have

$$
h_{x, d, x}=h_{x, y, z^{-1}} ; \quad \text { see Corollary [5.8, }
$$

By (P4), we have $\mathbf{a}(x)=\mathbf{a}\left(z^{-1}\right)$. Hence the above identity implies that

$$
\begin{aligned}
\gamma_{x, y, z} & =\text { constant term of } v^{\mathbf{a}\left(z^{-1}\right)} h_{x, y, z^{-1}} \\
& =\text { constant term of } v^{\mathbf{a}(x)} h_{x, d, x} \\
& =\gamma_{x, d, x^{-1}}=n_{d},
\end{aligned}
$$

as desired. 
Finally, we show that $n_{d}=1$ for any $d \in \mathcal{D}$. Let $\lambda \in \Lambda_{n}$ be such that $d \in \mathfrak{R}(\lambda)$ and write $d=\mathbf{w}_{\lambda}(j, j)$ where $1 \leqslant j \leqslant d_{\lambda}$. We consider the element $x_{j} w_{\lambda}=\mathbf{w}_{\lambda}(j, 1)$ where $x_{j} \in X_{\lambda}$; see Remark 3.9. Using the description of $C_{w_{\lambda}}$ in Lemma 2.8 and the multiplication rules in (2.2), one easily shows that

Thus, we have

$$
C_{x_{j} w_{\lambda}} C_{w_{\lambda}}=\varepsilon_{w_{\lambda}} v^{-l\left(w_{\lambda}\right)} P_{\lambda} C_{x_{j} w_{\lambda}}
$$

$$
h_{x_{j} w_{\lambda}, w_{\lambda}, x_{j} w_{\lambda}}=v^{-l\left(w_{\lambda}\right)} P_{\lambda} .
$$

Now, by Remark [3.5, we have $x_{j} w_{\lambda} \sim_{\mathcal{R}} d$. Furthermore, since $w_{\lambda} x_{j}^{-1}=$ $\left(x_{j} w_{\lambda}\right)^{-1}=\mathbf{w}_{\lambda}(1, j)$, we have $w_{\lambda} \sim_{\mathcal{R}} w_{\lambda} x_{j}^{-1} \sim_{\mathcal{L}} d$. Hence Corollary 5.8 implies that

$$
h_{x_{j} w_{\lambda}, w_{\lambda} x_{j}^{-1}, d}=h_{x_{j} w_{\lambda}, w_{\lambda}, x_{j} w_{\lambda}}=v^{-l\left(w_{\lambda}\right)} P_{\lambda} .
$$

By (P4), we have $\mathbf{a}(d)=\mathbf{a}\left(x_{j} w_{\lambda}\right)$ and this equals $a(\lambda)=l\left(w_{\lambda}\right)$, as we have seen at the beginning of the proof. Since $P_{\lambda}$ is a polynomial with constant term 1 , we now conclude that

$$
\gamma_{x_{j} w_{\lambda}, w_{\lambda} x_{j}^{-1}, d}=\gamma_{x_{j} w_{\lambda}, w_{\lambda}, w_{\lambda} x_{j}^{-1}}=1 .
$$

By (P5), the left hand side equals $n_{d}$. Thus, $n_{d}=1$.

We can now also determine the sign in Theorem 4.10 and Corollary 4.11

Corollary 5.11. We have $\eta_{w}=1$ for all $w \in \mathfrak{S}_{n}$.

Proof. Let $\lambda \in \Lambda_{n}$ be such that $w \in \mathfrak{R}(\lambda)$ and write $w=\mathbf{w}_{\lambda}(i, j)$ where $1 \leqslant i, j \leqslant d_{\lambda}$. Then we have

$$
\varepsilon_{w_{\lambda}} P_{\lambda} Z_{w}=v^{l\left(w_{\lambda}\right)} C_{x_{i} w_{\lambda}} C_{w_{\lambda} x_{j}^{-1}}=\sum_{z \in \mathfrak{S}_{n}} \varepsilon_{x_{i}} \varepsilon_{x_{j}} \varepsilon_{z} v^{l\left(w_{\lambda}\right)} h_{x_{i} w_{\lambda}, w_{\lambda} x_{j}^{-1}, z} C_{z} .
$$

On the other hand, by Theorem 4.10(a), we have $Z_{w} \equiv \eta_{w} C_{w} \bmod \hat{\mathcal{N}}^{\lambda}$ where $\eta_{w}= \pm 1$. Using Theorem 4.10(b), we conclude that

$$
P_{\lambda} \varepsilon_{w_{\lambda}} \eta_{w}=\varepsilon_{x_{i}} \varepsilon_{x_{j}} \varepsilon_{w} v^{l\left(w_{\lambda}\right)} h_{x_{i} w_{\lambda}, w_{\lambda} x_{j}^{-1}, w} .
$$

By Theorem 5.10, we have $\mathbf{a}(w)=a(\lambda)=l\left(w_{\lambda}\right)$. Hence, taking constant terms on both sides of the above identity, we obtain

$$
\eta_{w}=\varepsilon_{x_{i}} \varepsilon_{x_{j}} \varepsilon_{w} \varepsilon_{w_{\lambda}} \gamma_{x_{i} w_{\lambda}, w_{\lambda} x_{j}^{-1}, w^{-1}}
$$

Now, in Theorem 5.10, we also have seen that the constants $\gamma_{x, y, z}$ are either 0 or 1 . This yields that

$$
\eta_{w}=\varepsilon_{x_{i}} \varepsilon_{x_{j}} \varepsilon_{w_{\lambda}} \varepsilon_{w}
$$

Now let $\mathfrak{C}$ be the left cell containing $w_{\lambda}$ and $\mathfrak{C}_{1}$ be the left cell containing $w_{\lambda} x_{j}^{-1}$. Then we have a bijection $\mathfrak{C} \stackrel{\sim}{\rightarrow} \mathfrak{C}_{1}$ as in Corollary 5.8. Under this bijection, $w_{\lambda} \in \mathfrak{C}$ corresponds to $w_{\lambda} x_{j}^{-1} \in \mathfrak{C}_{1}$, and $x_{i} w_{\lambda} \in \mathfrak{C}$ corresponds to $w \in \mathfrak{C}_{1}$. Since that bijection is the composition of a finite number of star 
operations, a correspondingly repeated application of the relation in (2.6) (d) yields

$$
l\left(w_{\lambda}\right)+l\left(x_{i} w_{\lambda}\right) \equiv l\left(w_{\lambda} x_{j}^{-1}\right)+l(w) \quad \bmod 2 .
$$

Consequently, we have $\eta_{w}=1$, as desired.

Acknowledgements. This paper was written while the author enjoyed the hospitality of the Bernoulli center at the EPFL Lausanne (Switzerland), in the framework of the research program "Group representation theory" from january to june 2005 .

\section{REFERENCES}

[1] S. ARIKI, Robinson-Schensted correspondence and left cells. Combinatorial methods in representation theory (Kyoto, 1998), 1-20, Adv. Stud. Pure Math., 28, Kinokuniya, Tokyo, 2000.

[2] R. Dipper and G. D. James, Representations of Hecke algebras of general linear groups, Proc. London Math. Soc. 52 (1986), 20-52.

[3] R. Dipper and G. D. James, Blocks and idempotents of Hecke algebras of general linear groups, Proc. London Math. Soc. 54 (1989), 57-82.

[4] J. Du, B. Parshall, And L. Scott, Cells and $q$-Schur algebras, Transformation Groups 3 (1998), 33-49.

[5] M. GEck, Constructible characters, leading coefficients and left cells for finite Coxeter groups with unequal parameters, Represent. Theory 6 (2002), 1-30 (electronic).

[6] M. GECK, On the induction of Kazhdan-Lusztig cells, Bull. London Math. Soc. 35 (2003), 608-614.

[7] M. GECK AND L. IANCU, Lusztig's $a$-function in type $B_{n}$ in the asymptotic case, preprint (april 2005); available at http://arXiv.org/math.RT/0504213

[8] M. Geck And G. Pfeiffer, Characters of finite Coxeter groups and Iwahori-Hecke algebras, London Math. Soc. Monographs, New Series 21, Oxford University Press, 2000.

[9] P. N. Hoefsmit, Representations of Hecke algebras of finite groups with BN pairs of classical type, Ph.D. thesis, University of British Columbia, Vancouver, 1974.

[10] D. Kazhdan and G. Lusztig, Representations of Coxeter groups and Hecke algebras, Invent. Math. 53 (1979), 165-184.

[11] D. E. KNUTh, The art of computer programming, volume 3: Sorting and Searching, Addison-Wesley, Second Edition, 1998.

[12] G. Lusztig, On a theorem of Benson and Curtis, J. Algebra 71 (1981), 490-498.

[13] G. Lusztig, Characters of reductive groups over a finite field, Annals Math. Studies 107 (Princeton University Press, 1984).

[14] G. Lusztig, Cells in affine Weyl groups, Advanced Studies in Pure Math. 6, Algebraic groups and related topics, Kinokuniya and North-Holland, 1985, 255-287.

[15] G. Lusztig And N. Xi, Canonical left cells in affine Weyl groups, Advances in Math. 72 (1988), 284-288.

[16] G. LuszTig, Intersection cohomology methods in representation theory, Proceedings of the International Congress of Mathematics, Kyoto, Japan, 1990 (I. Satake, ed.), Springer-Verlag, 1991, pp. 155-174.

[17] G. Lusztig, Hecke algebras with unequal parameters, CRM Monographs Ser. 18, Amer. Math. Soc., Providence, RI, 2003.

[18] A. Mathas, Iwahori-Hecke algebras and Schur algebras of the symmetric group, Univ. Lecture Ser., vol. 15, Amer. Math. Soc., Providence, RI, 1999. 
[19] T. P. McDonough and C. A. Pallikaros, On relations between the classical and the Kazhdan-Lusztig representations of symmetric groups and associated Hecke algebras, preprint (2004).

[20] G. E. Murphy, On the representation theory of the symmetric groups and associated Hecke algebras, J. Algebra 152 (1992), 492-513.

[21] G. E. Murphy, The representations of Hecke algebras of type $A_{n}$, J. Algebra 173 (1995), 97-121.

[22] T. A. Springer, Quelques applications de la cohomologie d'intersection, Séminare Bourbaki (1981/82), exp. 589, Astérisque 92-93 (1982).

Institut Girard Desargues, bat. Jean Braconnier, Université Lyon 1, 21 aV Claude Bernard, F-69622 Villeurbanne Cedex, France

E-mail address: geck@igd.univ-lyon1.fr 